\title{
FORMAL DEFORMATION OF CHOW GROUPS
}

\author{
MARK GREEN* AND PHILLIP GRIFFITHS
}

\section{OutLine}

(1) Introduction

(i) Summary of results

(ii) Discussion of techniques

(2) First order obstruction theory

(i) Variants of Kodaira-Spencer theory

(ii) First order obstructions to lifting Chow groups

(3) Higher order obstruction theory

(i) Higher order Kodaira-Spencer theory

(ii) Higher order obstructions to lifting Chow groups; applications

(4) Failures of the formal theory

\section{InTRODUCTION}

(i) Summary of results.

The problem of constructing algebraic cycles with given properties - e.g., in a given homology class - is one of the longest standing and deepest questions in algebraic geometry. Over the years there have been several variational approaches centered around the question

(1(i).1) Given a family $\left\{X_{t}\right\}_{t \in B}$ of smooth algebraic varieties with $X_{t_{0}}=X$, and given a codimension- $p$ algebraic subvariety $Z \subset X$ whose fundamental class $\psi_{0}(Z)$ remains of Hodge type $(p, p)$ in $H^{2 p}\left(X_{t}, \mathbb{C}\right)$ for $t$ in an analytic neighborhood of $t_{0}$, is there a family of subvarieties $\left\{Z_{t}\right\}_{t \in B}$ with $Z_{t_{0}}=Z$ ?

Especially notable here is Spencer Bloch's semi-regularity paper [B]. The advantage of this approach is that one can use the full strength of the deformation theory of subvarieties. One might similarly ask whether there is a family of algebraic cycles $\left\{Z_{t}\right\}_{t \in B}$ deforming $Z$; some first steps toward an infinitesimal theory for this problem were the subject of [G-G2]. A third possible context, which we will consider here, is to work in the Chow group. Thus, letting $\xi \in C H^{p}(X)$ be the

*Research partially supported by NSF grant DMS 9970307. 
rational equivalence class represented by $[Z]$, the question (1(i).1) should be first modified to

(1(i).2) Under the assumptions in (1(i).1), is there a family $\xi_{t} \in C H^{p}\left(X_{t}\right)$ with $\xi_{t_{0}}=\xi$ ?

There is a basic obstruction to solving this problem, namely that the fundamental class $\psi_{0}(\xi)$ must remain of Hodge type $(p, p)$ in $H^{2 p}\left(X_{t}, \mathbb{C}\right)$ for $t$ in an analytic neighborhood of $t_{0}$. For $p=1$, this is the only obstruction. We will see below, for $p \geq 2$ that even modulo torsion there are other obstructions to deforming $\xi$ even when $\psi_{0}(\xi)=0$. The full question (1(i)2) is interesting, as is the question

(1(i).3) Under the assumptions of (1(i).1), is there a family $\xi_{t} \in C H^{p}\left(X_{t}\right)$ with

$$
\psi_{0}\left(\xi_{0}\right)=\psi_{0}(\xi) ?
$$

This question has a formal analogue where, so to speak, we try to construct $\xi_{t}$ as a formal power series in $t$, and the purpose of this paper is to develop an obstruction calculus that will help to understand the formal analogue of (1(i).3). Two applications of this calculus are the following, where again everything is modulo torsion

(1(i).4) Suppose that $\xi \in C H^{p}(X)$ and as in (1(i).1) assume that the fundamental class $\psi_{0}(\xi)$ remains of Hodge type $(p, p)$ for $t$ in an analytic neighborhood of $t_{0}$. Suppose further that

$$
H^{p+1}\left(\Omega_{X / \mathbb{C}}^{p-2}\right)=\cdots=H^{p+1}\left(\mathcal{O}_{X}\right)=0 .
$$

Then (1(i).2) is true formally.

For $p=2$, we are hypothesizing that

$$
H^{3}\left(\mathcal{O}_{X}\right)=0
$$

which is equivalent to saying

$$
J^{2}(X)=J^{2}(X)_{a b},
$$

i.e. $J^{2}(X)$ is an abelian variety. By upper-semicontinuity the same should be true for nearby $X_{t}$. By the Generalized Hodge Conjecture, all of the $J\left(X_{t}\right)$ should lie in the image of the Abel-Jacobi maps, and we expect no further obstructions beyond $\psi_{0}(\xi)$ remaining of Hodge type. However, if

$$
H^{3}\left(\mathcal{O}_{X}\right) \neq 0
$$

then there exist cycles $\xi$ with $\psi_{0}(\xi)=0$ and $A J_{X}^{2}(\xi)$ non-torsion, yet where

$$
\operatorname{im}\left(A J_{X_{t}}^{2}\right) \subseteq \text { torsion for } 0<|t|<\epsilon
$$

as in $[\mathrm{G}]$ and $[\mathrm{V}]$. So (1(i).2) cannot hold in this situation. The precise explanation of what "being true formally" means will be given below. We will also see that the assumption (1(i).5) implies that, at least formally, questions (1(i).2) and (1(i).3) coincide; i.e., there are no obstructions to formally lifting $F^{1} C H^{p}(X)$. 
In general we will see that:

(1(i).6) If

$$
H^{p+1}\left(\Omega_{X / \mathbb{C}}^{p-m-1}\right)=\cdots=H^{p+1}\left(\mathcal{O}_{X}\right)=0, \quad m \geqq 1,
$$

then $F^{m} C H^{p}(X)$ is formally unobstructed to all orders in $t$. Taking $m=$ $p$ we have that

$F^{p} C H^{p}(X)$ is formally unobstructed.

Here we use the version of the conjectural filtration on Chow groups of [G-G1].

Since the fundamental works of Bloch and others (cf. [B], [Q] and [S]) leading to the identification, again modulo torsion,

$$
C H^{p}(X) \otimes \mathbb{Q} \cong H^{p}\left(\mathcal{K}_{p}^{M}\left(\mathcal{O}_{X}\right)\right) \otimes \mathbb{Q}
$$

where $\mathcal{K}_{p}^{M}\left(\mathcal{O}_{X}\right)$ is the sheaf associated to the $p^{\text {th }}$-Milnor $K$-groups of the local rings $\mathcal{O}_{X, x}$ for some time it has been understood that for $p \geqq 2$ the groups $C H^{p}(X)$ have an arithmetic aspect. Below we shall show that:

(1(i).9) Suppose that $X$ is defined over $\mathbb{Q}$ and that $\xi \in C H^{p}(X(\mathbb{Q}))$ and, as in $(1(i) .4), \psi_{0}(\xi)$ remains of Hodge type $(p, p)$ for $t$ in an analytic neighborhood of $t_{0}$. Then formally (1(i).2) has an affirmative answer.

We shall also see that, in general, the lifting of $\xi \in C H^{p}(X(\mathbb{Q}))$ can only be formal. Of course, if the $\mathrm{HC}$ is true then question (1(i).3) will have an affirmative answer. Thus, there is some arithmetic/geometric subtlety in the difference between (1(i).2) and (1(i).3).

Before turning to a more detailed description of the contents of this paper we might summarize by saying that this work contains good news and bad news: The good news is that, under only Hodge-theoretic assumptions, we are able to construct something; the bad news is that there are subtle obstructions to the convergence of this iterative construction. There has been a significant recent progress in tackling such issues, cf. the excellent survey of Chambert-Loir [C-L].

(ii) Discussion of techniques

We will work with an algebraic family of algebraic varieties, given by

$$
X \stackrel{\pi}{\rightarrow} B \text {. }
$$

Here, $X$ and $B$ are smooth complex varieties and $\pi$ is a smooth projective map. We shall sometimes write (1(ii).1) as

$$
\left\{X_{t}\right\}_{t \in B}
$$

where $X_{t}=\pi^{-1}(t)$ is a smooth, projective variety. It will cause no essential loss in generality if we assume that $B$ is one-dimensional. We let $t_{0} \in B$ be a reference point and set $X=X_{t_{0}}$; we then may think of (1(ii).1) as a deformation of $X$. We denote by $\mathcal{J}_{X} \subset \mathcal{O}_{X}$ the ideal sheaf of $X_{t_{0}}$ and set

$$
\left\{\begin{aligned}
\mathcal{O}_{X_{m}} & =\mathcal{O}_{X / \mathcal{J}_{X}^{m+1}} \\
X_{m} & =\text { scheme }\left(X_{t_{0}}, \mathcal{O}_{X_{m}}\right) .
\end{aligned}\right.
$$


Geometrically the scheme $X_{m}$ is the $m^{\text {th }}$ order neighborhood of $X$ in $X$, or equivalently the deformation to order $m$ of $X$ given by (1(ii).2). We have natural maps

$$
x_{m+1} \longrightarrow x_{m}
$$

and we let

$$
\hat{x}=\lim _{m} x_{m}
$$

be the scheme $\left(X, \mathcal{O}_{\hat{x}}\right)$ where $\mathcal{O}_{\hat{x}, x}=\lim _{m} \mathcal{O}_{X_{m}}$; we shall refer to $\hat{X}$ as the formal neighborhood of $X$ in $X$.

For a codimension- $p$ algebraic cycle $Z$ we denote by $\xi=[Z] \in C H^{p}(X)$ the corresponding rational equivalence class. Throughout this paper we will work modulo torsion. We denote by

$$
\psi_{0}(\xi)=\psi_{0}(Z) \in H g^{p}(X)
$$

the fundamental class of $\xi$ or $Z$. Here

$$
H g^{p}(X)=H^{2 p}(X, \mathbb{Z}) \cap H^{p, p}(X)
$$

is the usual Hodge group of integral cohomology classes of Hodge type $(p, p)$, considered modulo torsion.

Fundamental to this work is the Soulé variant of the Bloch-Quillen identification

$$
C H^{p}(X) \cong H^{p}\left(\mathcal{K}_{p}^{M}\left(\mathcal{O}_{X}\right)\right) \quad \bmod \text { torsion . }
$$

Relative to a Zariski covering $U_{\alpha}$ of $X$, we may represent elements on the right as cocycles formed from symbols

$$
\left\{f_{1}, \ldots, f_{p}\right\}, \quad f_{i} \in \mathcal{O}_{X}^{*}\left(U_{\alpha_{0}} \cap \cdots \cap U_{\alpha_{p}}\right) .
$$

If $Z$ is a codimension- $p$ subvariety whose ideal sheaf has a resolution by locally free sheaves, then from this data we may construct the corresponding cocycle (1(ii).3). Thus, in a sense we may think of the RHS as giving the "equations" of the rational equivalence class of an algebraic cycle.

The central thrust of this work is to develop a calculus for successively lifting a class $\xi \in H^{p}\left(\mathcal{K}_{p}^{M}\left(\mathcal{O}_{X}\right)\right)$ to classes $\xi_{m}, \in H^{p}\left(\mathcal{K}_{p}^{M}\left(\mathcal{O}_{X_{m}}\right)\right)$ over the infinitesimal neighborhoods of $X$ in $X$. We are not saying that the identification (1(i).8) extends to the schemes $X_{m}$ (this may be an interesting question). Rather, if we can lift $\xi=[Z]$ to a class in $H^{p}\left(U, \mathcal{K}_{p}^{m}\left(\mathcal{O}_{U}\right)\right)$ where $U$ is a Zariski neighborhood of $X$ in $X$, then from

$$
C H^{p}(U) \cong H^{p}\left(U, \mathcal{K}_{p}^{M}\left(\mathcal{O}_{U}\right)\right)
$$

we will have lifted $[Z]$ to $[Z] \in C H^{p}(U)$ where $Z$ is a codimension- $p$ algebraic cycle in $U$. Our work will enable us to analyze the corresponding formal question of lifting $\xi$ to $\hat{\xi} \in H^{p}\left(\mathcal{K}_{p}^{M}\left(\mathcal{O}_{\hat{x}}\right)\right)$.

The general mechanisms for lifting geometric objects given by "equations" over successive infinitesimal neighborhoods is well known and classical, dating from the earliest works of Kodaira and Spencer. The fundamental observation in the present study is the following: Recall that for any ring $R$ there is a natural map

$$
K_{p}^{M}(R) \longrightarrow \Omega_{R / \mathbb{Q}}^{p}
$$

where the RHS are the absolute Kähler differentials. The map (1(ii).4) is induced by

$$
\left\{r_{1}, \ldots, r_{p}\right\} \longrightarrow \frac{d r_{1}}{r_{1}} \wedge \cdots \wedge \frac{d r_{p}}{r_{p}}, \quad r_{i} \in R^{*}
$$

By abuse of notation we denote this $d \log r_{1} \wedge \cdots \wedge d \log r_{p}$. 
For any scheme $Y$ the maps (1(ii).4) give maps

$$
\mathcal{K}_{p}^{M}\left(\mathcal{O}_{Y}\right) \longrightarrow \Omega_{Y / \mathbb{Q}}^{p}
$$

inducing on cohomology what is called the arithmetic cycle class mapping (cf. [Sv])

$$
\eta: H^{p}\left(\mathcal{K}_{p}^{M}\left(\mathcal{O}_{Y}\right)\right) \longrightarrow H^{p}\left(\Omega_{Y / \mathbb{Q}}^{p}\right)
$$

The basic observation in this work is:

(1(ii).6) The obstruction to lifting $\xi_{m} \in H^{p}\left(\mathcal{K}_{p}^{M}\left(\mathcal{O}_{X_{m}}\right)\right)$ to $H^{p}\left(\mathcal{K}_{p}^{M}\left(\mathcal{O}_{X_{m+1}}\right)\right)$ depends only on the arithmetic cycle class $\eta\left(\xi_{m}\right) \in H^{p}\left(\Omega_{x_{m} / \mathbb{Q}}^{p}\right)$.

To interpret (1(ii).6) we recall from [G-G1] that, assuming the important conjecture of Bloch-Beilinson, the arithmetic cycle class $\eta(Z)$ captures some - but not all — of the information in $[Z] \in C H^{p}(X)$. For $p=1$ this "lost" information is not crucial to the obstruction to lifting $\xi=[Z]$, this being essentially due to the $p=1$ case of (1(i).7). However, for $p \geqq 2$ this lost information is non-trivial for the lifting problem and may be related to the issue of non-convergence of the formal iterative construction leading to the results (1(i).4) and (1(i).9).

In section 2(i) we will review the classical $1^{\text {st }}$ order Kodaira-Spencer theory, especially the extension class and subsequent coboundary maps associated to the exact sheaf sequence

$$
0 \longrightarrow \Omega_{X / \mathbb{Q}}^{p-1} \oplus \Omega_{X / \mathbb{Q}}^{p} \longrightarrow \Omega_{x_{1 / \mathbb{Q}}}^{p} \longrightarrow \Omega_{X / \mathbb{Q}}^{p} \longrightarrow 0
$$

where the first map is

$$
\varphi \oplus \omega \mapsto d t \wedge \varphi+t \omega, \quad t^{2}=0 .
$$

The central fact, deriving ultimately from Kähler geometry, is that the coboundary map in the exact cohomology sequence of (1(ii).7)

$$
H^{q}\left(\Omega_{X / \mathbb{Q}}^{p}\right) \stackrel{\delta}{\longrightarrow} H^{q+1}\left(\Omega_{X / \mathbb{Q}}^{p-1}\right),
$$

is zero. We also establish another result (proposition (2(i).13)) that follows from the degeneracy at $E_{2}$ of the Leray spectral sequence associated to a smooth, projective morphism.

In section 2(ii) we analyze the $1^{\text {st }}$ order obstruction theory for the groups $H^{p}\left(\mathcal{K}_{p}^{M}\left(\mathcal{O}_{X}\right)\right)$. The basic observation is the commutative diagram

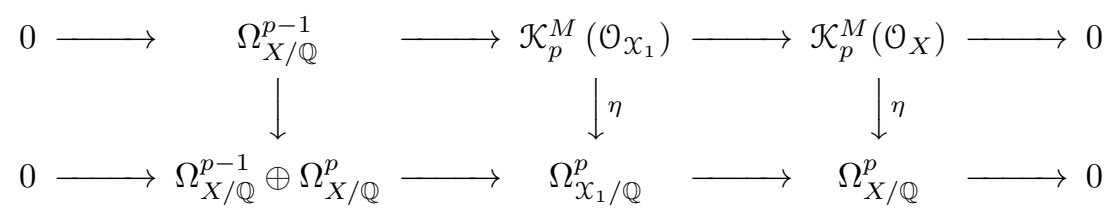

where the left hand vertical arrow is

$$
\varphi \longrightarrow \varphi \oplus d \varphi
$$

and the top left hand horizontal arrow is induced by

$$
f \frac{d g_{1}}{g_{1}} \wedge \cdots \wedge \frac{d g_{p-1}}{g_{p-1}} \mapsto\left\{1+t f, g_{1}, \ldots, g_{p-1}\right\}, \quad t^{2}=0
$$


where $f \in \mathcal{O}_{X, x}, g_{i} \in \mathcal{O}_{X, x}^{*}$. The $m=1$ result (1(ii).6) follows from (1(ii).8) and (1(ii).9). The geometric understanding of the relationship between first order obstruction theory and the filtration on $H^{p}\left(\mathcal{K}_{p}^{M}\left(\mathcal{O}_{X}\right)\right) \cong C H^{p}(X)$ is also given.

In section 3(i) we give the rudiments of higher order Kodaira-Spencer theory in a form that is amenable to the calculations we need to make. (We make no attempt to provide a systematic general theory as is done in $[\mathrm{R}]$ and $[\mathrm{E}-\mathrm{V}]$.) In particular the higher order analogues of (1(ii).7) and (1(ii).8) are given.

Finally in section 3(ii) we turn to the higher order deformation for the groups $H^{p}\left(\mathcal{K}_{p}^{M}\left(\mathcal{O}_{X}\right)\right)$. The analogue of (1(ii).9) is the diagram

(1(ii).11)

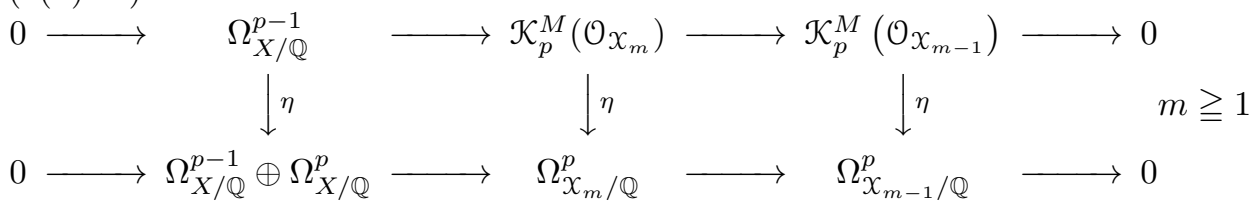

where the left hand map in the top row is the same as (1(ii).10) with $t^{m}$ replacing $t$; the exactness here requires a somewhat intricate lemma on $\epsilon$-Steinberg symbols (cf. (3(ii).3)). With this formalism in place we give the proofs of the main applications (1(i).5)-(1(i).7) and (1(i).9).

In Section 4 we discuss failures of the formal theory; i.e., examples where all the obstructions to formally lifting vanish but where no choice of successive extensions will lead to a convergent, or geometric, result. This phenomenon was encountered in an earlier work [G-G1], and it may have to do with theory of $G$-functions (cf. $[\mathrm{A}])$. We feel that its understanding presents a significant issue.

\section{First ORDER OBSTRUCTION THEORY}

\section{(i) Variants of Kodaira-Spencer theory}

In this section, we collect together some classical results of deformation theory. Given a family $X \stackrel{\pi}{\longrightarrow} B$, the basic object in classical Kodaira-Spencer theory is the exact sequence

$$
0 \longrightarrow \Theta_{X / B} \longrightarrow \Theta_{X} \longrightarrow \pi^{*} \Theta_{B} \longrightarrow 0 \text {. }
$$

Letting $t$ denote a local uniformizing parameter on $B$ with $\pi^{-1}\left(t_{0}\right)=X$, we may rewrite this sequence as

$$
0 \longrightarrow \Theta_{x / B} \longrightarrow \Theta_{x} \longrightarrow \mathcal{O}_{x} \otimes \partial / \partial t \longrightarrow 0
$$

Reducing this sequence modulo $t^{m+1}$ and setting

$$
\Theta_{m}=\Theta_{X / B} /\left(t^{m+1}\right)
$$

we obtain

$$
0 \longrightarrow \Theta_{m} \longrightarrow \Theta_{x_{m}} \longrightarrow \mathcal{O}_{x_{m}} \otimes \partial / \partial t \longrightarrow 0 \text {. }
$$

The $1^{\text {st }}$ order classical Kodaira-Spencer theory is derived from the $m=0$ case of this sequence, which setting $\Theta_{0}=\Theta_{X}$ we write as

$$
0 \longrightarrow \Theta_{X} \longrightarrow \Theta_{x_{1}} \longrightarrow \mathcal{O}_{X} \otimes \partial / \partial t \longrightarrow 0 \text {. }
$$


Denoting by $\delta$ the coboundary map in exact cohomology sequences we have the classical

Definition: The $\left(1^{\text {st }}\right.$ order $)$ Kodaira-Spencer class associated to $X \longrightarrow B$ is given by

$$
\theta_{1}=\delta(\partial / \partial t) \in H^{1}\left(\Theta_{X}\right) .
$$

Geometrically, we think of $\theta_{1}$ as the obstruction to lifting $\partial / \partial t$ to the fibre over $t=0$ in the family $X \longrightarrow B$. This is clearly the obstruction to trivializing $X \longrightarrow B$ to $1^{\text {st }}$ order, i.e.,

$$
\theta_{1}=0 \Leftrightarrow X_{1} \cong X \times \operatorname{Spec}\left(\mathbb{C}[t] / t^{2}\right) .
$$

Equivalently, $\theta_{1}$ is the obstruction to splitting the sequence given by (2(i).2), this obstruction being the same as the extension class in $H^{1}\left(\operatorname{Hom}\left(\mathcal{O}_{X}, \Theta\right)\right) \otimes \partial / \partial t \cong$ $H^{1}\left(\Theta_{X}\right)$ of $(2(\mathrm{i}) \cdot 2)$.

When we study the geometry of $X \longrightarrow B$ inductively over the successive infinitesimal neighborhoods $X_{k}$, especially in the study of algebraic cycles and cohomology, there are a number of related sequences to (2(i).1). We shall now discuss their $1^{\text {st }}$ order versions.

On the $1^{\text {st }}$ order neighborhood $X_{1}$, we have $t^{2}=0$ and the three basic sequences for our study are

$$
\begin{cases}\text { (i) } & 0 \longrightarrow \mathcal{O}_{X} \longrightarrow \mathcal{O}_{X_{1}} \longrightarrow \mathcal{O}_{X} \longrightarrow 0 \\ \text { (ii) } & 0 \longrightarrow \mathcal{O}_{X} \oplus \Omega_{X / \mathbb{C}}^{1} \longrightarrow \Omega_{X_{1} / \mathbb{C}}^{1} \longrightarrow \Omega_{X / \mathbb{C}}^{1} \longrightarrow 0 \\ \text { (iii) } & 0 \longrightarrow \Theta_{X} \longrightarrow \Theta_{X_{1}} \longrightarrow \pi^{*} \Theta_{B} \longrightarrow 0\end{cases}
$$

Here, the right arrows are restriction mappings and the left arrows are given respectively by

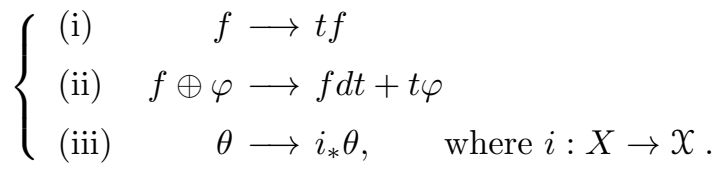

More precisely, under (i) we have to give for each $x \in X$ a map

$$
\mathcal{O}_{X, x} \stackrel{t}{\longrightarrow} \mathcal{O}_{X_{1}, x} .
$$

For $f \in \mathcal{O}_{X, x}$ we choose any $\tilde{f} \in \mathcal{O}_{X_{1}, x}$ with $\left.\tilde{f}\right|_{X}=f$ and then the map is

$$
f \longrightarrow t \tilde{f}
$$

Since $t^{2}=0$, the choice of lifting $\tilde{f}$ does not matter. Similar remarks apply to (ii) and (iii). We may write

$$
\left\{\begin{array}{l}
\text { (i) }=t \\
\text { (ii) }=d t \oplus t \\
\text { (iii) }=t .
\end{array}\right.
$$

The extension classes of the sequences (2(i).3) are of interest. By the remarks above we expect that all of these can be derived from the $1^{\text {st }}$ order Kodaira-Spencer class $\theta_{1} \in H^{1}\left(\Theta_{X}\right)$, and we shall now explain how this goes. For this we note that 
there are pairings

$$
\begin{cases}\Theta_{X} \otimes_{\mathbb{C}} \mathcal{O}_{X} & \longrightarrow \mathcal{O}_{X} \\ \Theta_{X} \otimes_{\mathbb{C}} \Omega_{X / \mathbb{C}}^{1} & \longrightarrow \mathcal{O}_{X} \oplus \Omega_{X / \mathbb{C}}^{1} \\ \Theta_{X} \otimes_{\mathbb{C}} \Theta_{X} & \longrightarrow \Theta_{X}\end{cases}
$$

given respectively by

$$
\begin{cases}\theta \otimes f \longrightarrow \mathcal{L}_{\theta} f & (=\theta \cdot f) \\ \theta \otimes \omega \longrightarrow \theta\rfloor \omega \oplus \mathcal{L}_{\theta} \omega & \\ \theta \otimes \sigma \longrightarrow \mathcal{L}_{\theta} \sigma & (=[\theta, \sigma])\end{cases}
$$

where $\mathcal{L}$ denotes Lie derivative. Of these maps only $\theta \otimes \omega \longrightarrow \theta\rfloor \omega$ is $\mathcal{O}_{X}$-linear; the remainder are $\mathbb{C}$-linear derivations.

(2(i).5) Proposition: The extension classes associated to the exact sequences (2(i).3) are respectively

$$
\left\{\begin{array}{l}
\text { (i) } \mathcal{L}_{\theta_{1}} \in H^{1}\left(\operatorname{Hom}_{\mathbb{C}}\left(\mathcal{O}_{X}, \mathcal{O}_{X}\right)\right) \\
\text { (ii) } \left.\theta_{1}\right\rfloor \oplus \mathcal{L}_{\theta_{1}} \in H^{1}\left(\operatorname{Hom}_{\mathcal{O}_{X}}\left(\Omega_{X / \mathbb{C}}^{1}, \mathcal{O}_{X}\right)\right) \oplus H^{1}\left(\operatorname{Hom}_{\mathbb{C}}\left(\Omega_{X / \mathbb{C}}^{1}, \Omega_{X / \mathbb{C}}^{1}\right)\right) \\
\text { (iii) } \mathcal{L}_{\theta_{1}} \in H^{1}\left(\operatorname{Hom}_{\mathbb{C}}\left(\Theta_{X_{1} / B}, \Theta_{X}\right)\right) .
\end{array}\right.
$$

Proof: We shall prove the most interesting case (ii); a similar argument will apply to the other cases. We shall also work in the analytic topology; the algebraic case may be established by a similar argument, or alternatively the result may be deduced from GAGA. Finally, we shall carry out the computations when $\operatorname{dim} X=1$; the extension to the general case is only notationally more complicated.

We may cover $X$ by open sets $U_{\alpha}$ in which there are box coordinates $\left(z_{\alpha}, t\right)$. In the overlaps $U_{\alpha \beta}=: U_{\alpha} \cap U_{\beta}$ we will have

$$
z_{\alpha}=f_{\alpha \beta}\left(z_{\beta}, t\right)=f_{\alpha \beta}\left(z_{\beta}\right)+f_{\alpha \beta 1}\left(z_{\beta}\right) t+f_{\alpha \beta 2}\left(t_{\beta}\right) \frac{t^{2}}{2}+\cdots .
$$

We first claim that the Kodaira-Spencer class is represented by the cocycle

$$
\theta_{1 \alpha \beta}=f_{\alpha \beta 1}\left(z_{\beta}\right) \partial / \partial z_{\alpha} \in H^{1}\left(\left\{U_{\alpha}\right\}, \Theta_{X}\right) .
$$

To see this, we let $(\partial / \partial t)_{\alpha}$ be the lift of $\partial / \partial t$ in the $\left(z_{\alpha}, t\right)$ coordinate system. Then in $U_{\alpha \beta}$

$$
\left\{\begin{array}{l}
\left.\left((\partial / \partial t)_{\beta}-(\partial / \partial t)_{\alpha}\right)\right) \cdot t=0 \\
\left((\partial / \partial t)_{\beta}-(\partial / \partial t)_{\alpha}\right) \cdot z_{\alpha}=\left.\frac{\partial f_{\alpha \beta}\left(z_{\beta}, t\right)}{\partial t}\right|_{t=0}=f_{\alpha \beta 1}\left(z_{\beta}\right),
\end{array}\right.
$$

which is what is wanted.

We now let $e_{\alpha}$ be the product extension of a function or a differential form from $U_{\alpha} \cap X$ to $U_{\alpha}$. Then by (2(i).6) we have

$$
\left(e_{\beta}-e_{\alpha}\right) d z_{\alpha}=\partial_{z_{\beta}} f_{\alpha \beta}\left(z_{\beta}\right) d z_{\beta}+f_{\alpha \beta 1}\left(z_{\beta}\right) d t+\partial_{z_{\beta}} f_{\alpha \beta 1}\left(z_{\beta}\right) t \bmod t^{2}, t d t .
$$

The first term is $d z_{\alpha}$ expressed in the $z_{\beta}$-coordinate system. The second term is, by (2(i).7),

$$
\left.\theta_{\alpha \beta 1}\right\rfloor d z_{\alpha}
$$


and using the Lie derivative formula the third term is the coefficient of $d z_{\beta}$ in

$$
\mathcal{L}_{\theta_{\alpha \beta 1}} d z_{\alpha}
$$

expressed in the $z_{\beta}$-coordinate system.

From (2(i).3) (ii) ${ }_{1}$ we infer the exact sequence

$$
\text { (ii) })_{p} \quad 0 \longrightarrow \Omega_{X / \mathbb{C}}^{p-1} \oplus \Omega_{X / \mathbb{C}}^{p} \longrightarrow \Omega_{x_{1} / \mathbb{C}}^{p} \longrightarrow \Omega_{X / \mathbb{C}}^{p} \longrightarrow 0
$$

whose extension class is

$$
\left.\theta_{1}\right\rfloor \oplus \mathcal{L}_{\theta_{1}}
$$

where the first term is induced from the pairing

$$
\Theta_{X} \otimes \Omega_{X / \mathbb{C}}^{p} \longrightarrow \Omega_{X / \mathbb{C}}^{p-1}
$$

It is well-known and elementary that the coboundary maps in the long exact cohomology sequences associated to short exact sheaf sequences are given by cupproducts with the extension classes.

(2(i).8) Proposition: The coboundary maps in the long exact cohomology sequences arising from (2(i).3)(i) and (ii) $)_{p}$ corresponding to $\mathcal{L}_{\theta_{1}}$ are zero. Thus the only nonzero coboundary maps are

$$
H^{q}\left(\Omega_{X / \mathbb{C}}^{p}\right) \stackrel{\left.\theta_{1}\right\rfloor}{\longrightarrow} H^{q+1}\left(\Omega_{X / \mathbb{C}}^{p-1}\right) .
$$

Proof: Using the Lie derivative formula

$$
\left.\left.\mathcal{L}_{\theta} \omega=d(\theta\rfloor \omega\right)+\theta\right\rfloor d \omega,
$$

the proposition follows from the Kähler manifold fact that the mapping

$$
H^{q}\left(\Omega_{X / \mathbb{C}}^{p}\right) \stackrel{d}{\longrightarrow} H^{q}\left(\Omega_{X / \mathbb{C}}^{p+1}\right)
$$

induced by the exterior derivative is zero. Indeed, there is a commutative diagram

$$
\begin{array}{cc}
H^{q}\left(\Omega_{X / \mathbb{C}}^{p}\right) & d \\
\imath \| & H^{q}\left(\Omega_{X / \mathbb{C}}^{p+1}\right) \\
{ }^{p} & \imath \| \\
H_{\bar{\partial}}^{p, q}(X) & \stackrel{\partial}{\longrightarrow} H_{\bar{\partial}}^{p+1, q}(X)
\end{array}
$$

(since $d=\partial$ on $\Omega_{X / \mathbb{C}}^{p}$ ), and it is a Kähler fact that every class in $H_{\bar{\partial}}^{p, q}(X)$ is represented by a $\partial$-closed form (e.g., take the harmonic representative for a given Kähler metric).

Remarks: This result has the following elementary geometric meaning: Given $\omega \in H^{q}\left(\Omega_{X / \mathbb{C}}^{p}\right)$,

$$
\mathcal{L}_{\theta_{1}} \omega \in H^{q+1}\left(\Omega_{X / \mathbb{C}}^{p}\right)
$$

is the $1^{\text {st }}$ obstruction to finding a family $\omega_{t} \in H^{q}\left(\Omega_{X_{t} / \mathbb{C}}^{p}\right)$ with $\omega_{0}=\omega$. But from

$$
H^{r}\left(X_{t}, \mathbb{C}\right) \cong \underset{p+q=r}{\oplus} H^{q}\left(\Omega_{X_{t} / \mathbb{C}}^{p}\right)
$$

it follows that $\operatorname{dim} H^{q}\left(\Omega_{X_{t} / \mathbb{C}}^{p}\right)$ is constant and hence $\omega$ extends to a family $\omega_{t}$. 
The $1^{\text {st }}$ obstruction to extending $\theta_{1} \in H^{1}\left(\Theta_{X}\right)$ to $\theta_{1, t} \in H^{1}\left(\Theta_{X_{t}}\right)$ is given by

$$
\mathcal{L}_{\theta_{1}}\left(\theta_{1}\right)=\left[\theta_{1}, \theta_{1}\right]
$$

which is also zero, although the map

$$
H^{q}\left(\Theta_{X}\right) \stackrel{\mathcal{L}_{\theta_{1}}}{\longrightarrow} H^{q+1}\left(\Theta_{X}\right)
$$

is in general non-zero.

Remark: A result of Herb Clemens (cf. [C]) states that for $\theta \in H^{1}\left(\Theta_{X}\right)$ the map

$$
[\theta, \theta]: H^{q}\left(\Omega_{X / \mathbb{C}}^{p}\right) \stackrel{\lrcorner}{\longrightarrow} H^{q+2}\left(\Omega_{X / \mathbb{C}}^{p-1}\right)
$$

is zero. More generally, for $\theta_{i} \in H^{r_{i}}\left(\Theta_{X}\right), i=1,2$, the map

$$
\left[\theta_{1}, \theta_{2}\right]: H^{q}\left(\Omega_{X / \mathbb{C}}^{p}\right) \longrightarrow H^{q+r_{1}+r_{2}}\left(\Omega_{X / \mathbb{C}}^{p-1}\right)
$$

is zero. This follows from the standard differential-geometric formula

$$
\left.\left.\left.\left(\theta_{1}\right\rfloor \mathcal{L}_{\theta_{2}}-\mathcal{L}_{\theta_{2}}\left(\theta_{1}\right\rfloor\right)\right) \omega=\left[\theta_{1}, \theta_{2}\right]\right\rfloor \omega
$$

together with

$$
\mathcal{L}_{\theta_{i}}: H^{q}\left(\Omega_{X / \mathbb{C}}^{p}\right) \longrightarrow H^{q}\left(\Omega_{X / \mathbb{C}}^{p}\right)
$$

being the zero map as a consequence of

$$
\left.\left.\mathcal{L}_{\theta_{i}} \omega=\theta_{1}\right\rfloor d \omega+d\left(\theta_{i}\right\rfloor \omega\right)
$$

as above.

Corollary: If $X$ is a Calabi-Yau manifold then for $\theta_{i} \in H^{r_{1}}\left(\Theta_{X}\right)$,

$$
\left[\theta_{1}, \theta_{2}\right]=0 \text {. }
$$

Proof: This follows from

$$
H^{r_{1}+r_{2}}\left(\Theta_{X}\right) \cong H^{r_{1}+r_{2}}\left(\Omega_{X / \mathbb{C}}^{n-1}\right)
$$

and the commutativity of the diagram

$$
\begin{array}{ccc}
H^{r}\left(\Theta_{X}\right) \otimes H^{n-r}\left(\Omega_{X / \mathbb{C}}^{1}\right) & \longrightarrow & H^{n}\left(\mathcal{O}_{X}\right) \\
\imath \| & 2 \| \\
H^{r}\left(\Omega_{X / \mathbb{C}}^{n-1}\right) \otimes H^{n-r}\left(\Omega_{X / \mathbb{C}}^{1}\right) & \longrightarrow & H^{n}\left(\Omega_{X / \mathbb{C}}^{n}\right),
\end{array}
$$

using that the pairing in the bottom row is non-degenerate.

Corollary (Bogomolov): Calabi-Yau varieties are unobstructed.

Proof: This is a well-known consequence of the fact that

$$
H^{1}\left(\Theta_{X}\right) \otimes H^{1}(\Theta)_{X} \stackrel{[,]}{\longrightarrow} H^{2}\left(\Theta_{X}\right)
$$

is zero.

Finally we want to extend the above discussion to absolute differentials. Here we assume that $X \longrightarrow B$ is algebraic and we work in the algebraic setting - Zariski topology, sheaf of regular (in the algebraic sense) functions, etc. We will discuss$$
\text { (iv) } 1
$$$$
0 \longrightarrow \mathcal{O}_{X} \oplus \Omega_{X / \mathbb{Q}}^{1} \longrightarrow \Omega_{X_{1} / \mathbb{Q}}^{1} \longrightarrow \Omega_{X / \mathbb{Q}}^{1} \longrightarrow 0
$$ 
and the sequences derived from it by exterior algebra. Here, for any scheme $Y$ we denote by $\Omega_{Y / \mathbb{Q}}$ the sheaf of absolute Kähler differentials. From (2(i).3)(iv) ${ }_{1}$ we deduce a diagram

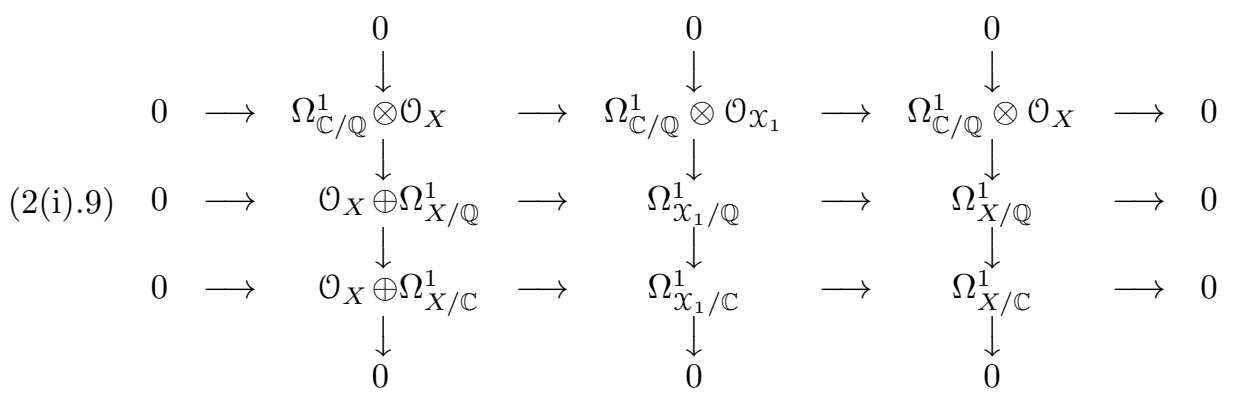

The left hand column is the same as the right hand column direct summed with $\mathcal{O}_{X}$ in the $\Omega_{X / \mathbb{Q}}^{1}$ and $\Omega_{X / \mathbb{C}}^{1}$ terms and with $\operatorname{id}_{\mathcal{O}_{X}}$ being the corresponding map.

The commutativity of the diagram (2(i).9) results from the following considerations. We let $k$ be a field of definition of $X$ and choose a smooth variety $S$ defined over $\mathbb{Q}$ and with

We then denote by

$$
k \cong \mathbb{Q}(S)
$$

$$
\mathcal{S}(X) \stackrel{\pi}{\longrightarrow} S
$$

the $k$-spread of $X$.

If $s_{0} \in S$ is a $\mathbb{Q}$-generic point of $S$, i.e., $s_{0}$ does not lie on any proper subvariety of $S$ defined over $\mathbb{Q}$, then

$$
\pi^{-1}\left(s_{0}\right) \cong X
$$

and under this identification,

$$
\left.\Omega_{X(k) / \mathbb{Q}}^{1} \cong \Omega_{\mathcal{S}(x)(\mathbb{Q}) / \mathbb{Q}}^{1}\right|_{\pi^{-1}\left(s_{0}\right)} .
$$

We also not that

$$
\left.\Omega_{k / \mathbb{Q}}^{1} \cong \Omega_{S(\mathbb{Q}) / \mathbb{Q}}^{1}\right|_{s_{0}}
$$

If $X \rightarrow B$ is defined over $k$, then for $t \in B(k)$ we have the $k$-spread

$$
\mathcal{S}\left(X_{t}\right) \rightarrow S
$$

of $X_{t}$. Passing to complex points, the family $\left\{\mathcal{S}\left(X_{t}\right)\right\}_{t \in B}$ gives

Replacing $\Omega_{\mathbb{C} / \mathbb{Q}}^{1}$ by $\Omega_{k / \mathbb{Q}}^{1}$ in the diagram (2(i).9) loses no geometric information. If

$$
X \stackrel{p}{\longrightarrow} B
$$

is a family defined over $k$, then we get

$$
\begin{gathered}
\mathcal{S}(X) \longrightarrow \mathcal{S}(B) \\
\searrow \swarrow \\
S .
\end{gathered}
$$


We may identify

$$
\left.\Omega_{X_{1}(k) / \mathbb{Q}}^{1} \cong \Omega_{\mathcal{S}(X)_{1}(\mathbb{Q}) / \mathbb{Q}}^{1}\right|_{\pi^{-1}\left(\mathcal{S}_{0}\right)} .
$$

The natural exact sequence

$$
0 \rightarrow \Omega_{k / \mathbb{Q}}^{1} \otimes \mathcal{O}_{X_{1}(k)} \rightarrow \Omega_{X_{1}(k) / \mathbb{Q}}^{1} \rightarrow \Omega_{X_{1}(k) / k}^{1} \rightarrow 0
$$

becomes

$$
\left.\left.\left.0 \rightarrow \Omega_{S(\mathbb{Q}) / \mathbb{Q})}^{1} \otimes \mathcal{O}_{\mathcal{S}(X)_{1}(\mathbb{Q}) / \mathbb{Q}}\right|_{s_{0}} \rightarrow \Omega_{\mathcal{S}(X)_{1}(\mathbb{Q}) / \mathbb{Q}}^{1}\right|_{s_{0}} \rightarrow \Omega_{\mathcal{S}(X)_{1} / S}^{1}\right|_{s_{0}} \rightarrow 0 .
$$

so that by base change the term in the middle in (2(i).9) may be thought of as the complexification of the 1 -forms in a $1^{\text {st }}$ order neighborhood of $X$ in $\mathcal{S}(X)$ and the horizontal and vertical arrows reflect the sequences of the type (2(i).3)(ii) $)_{1}$ in the $B$ and $S$ directions respectively.

Let $\sigma \in H^{1}\left(\Theta_{X(k)}\right) \otimes \Omega_{k / \mathbb{Q}}^{1}$ denote the $1^{\text {st }}$ order Kodaira-Spencer class of $\mathcal{S}(X) \rightarrow S$, where we identify $T_{s_{0}}^{*} S \cong \Omega_{k / \mathbb{Q}}^{1}$. Then $\sigma$ gives the extension class of

$$
0 \rightarrow \Omega_{k / \mathbb{Q}}^{1} \otimes \mathcal{O}_{X(k)} \rightarrow \Omega_{\mathcal{S}(X)(k)_{1} / \mathbb{Q}}^{1} \rightarrow \Omega_{X(k) / k}^{1} \rightarrow 0,
$$

and by the process described in proposition (2(i).5) it induces the extension classes of the vertical sequences in (2(i).9). Moreover we have

$$
\left[\theta_{1}, \sigma\right]=0
$$

and this implies the commutativity of (2(i).9).

For $\theta \in \Theta_{X(k)}$ the Lie derivative

$$
\mathcal{L}_{\theta}: \Omega_{X / \mathbb{Q}}^{1} \rightarrow \Omega_{X / \mathbb{Q}}^{1}
$$

may be defined by

$$
\left.\left.\mathcal{L}_{\theta}(\omega)=\theta\right\rfloor d \omega+d(\theta\rfloor \omega\right)
$$

where $d=d_{X / \mathbb{Q}}$. We will see that:

The induced map

is zero.

$$
\mathcal{L}_{\theta_{1}}: H^{q}\left(\Omega_{X / \mathbb{Q}}^{1}\right) \rightarrow H^{q+1}\left(\Omega_{X / \mathbb{Q}}^{1}\right)
$$

For general $p$, the sheaves $\Omega_{Y / \mathbb{Q}}^{p}$ are filtered by

$$
F^{m} \Omega_{Y / \mathbb{Q}}^{p}=\text { image }\left\{\Omega_{\mathbb{C} / \mathbb{Q}}^{m} \otimes \Omega_{Y / \mathbb{Q}}^{p-m} \rightarrow \Omega_{Y / \mathbb{Q}}^{p}\right\} .
$$

There is a spectral sequence which abuts to $H^{q}\left(\Omega_{Y / \mathbb{Q}}^{p}\right)$ and whose $E_{1}$-term is $\Omega_{\mathbb{C} / \mathbb{Q}}^{m} \otimes$ $H^{q}\left(\Omega_{Y / \mathbb{C}}^{p-m}\right)$. For $Y=X$ the maps $\mathcal{L}_{\theta_{1}}$ are zero on these groups and hence vanish on $H^{q}\left(\Omega_{X / \mathbb{Q}}^{p}\right)$. Thus we have the

(2(i).11) Proposition: The coboundary maps in the exact cohomology sequence of

$$
0 \rightarrow \Omega_{X / \mathbb{Q}}^{p-1} \oplus \Omega_{X / \mathbb{Q}}^{p} \rightarrow \Omega_{X_{1} / \mathbb{Q}}^{p} \rightarrow \Omega_{X / \mathbb{Q}}^{p} \rightarrow 0
$$

are $\left.\delta=\theta_{1}\right\rfloor \oplus \mathcal{L}_{\theta_{1}}$.

Theorem: $H^{q}\left(\Omega_{X / \mathbb{Q}}^{p}\right) \stackrel{\mathcal{L}_{\theta_{1}}}{\longrightarrow} H^{q+1}\left(\Omega_{X / \mathbb{Q}}^{p}\right)$ is 0 .

The proof proceeds by a number of steps.

(a) $\left.\Omega_{X(k) / \mathbb{Q}}^{p} \cong \Omega_{\mathcal{S}(X) / \mathbb{Q})}^{p}\right|_{s_{0}}$ where $s_{0}$ is a $\mathbb{Q}$-generic point of $S$. 
(b) Thus we need to show

$$
H^{q}\left(\left.\Omega_{\mathcal{S}(X)(\mathbb{Q}) / \mathbb{Q})}^{p}\right|_{s_{0}}\right) \stackrel{\mathcal{L}_{\theta_{1}}}{\longrightarrow} H^{q+1}\left(\left.\Omega_{\mathcal{S}(X)(\mathbb{Q}) / \mathbb{Q}}^{p}\right|_{s_{0}}\right)
$$

is zero.

(c)

$$
H^{q}\left(\left.\Omega_{\mathcal{S}(X)(\mathbb{Q}) / \mathbb{Q}}^{p}\right|_{s_{0}}\right) \cong \lim _{\begin{array}{c}
U \text { Zar open } \\
\text { def } / \mathbb{Q} \subseteq S
\end{array}} H^{q}\left(U, \Omega_{\mathcal{S}(X)(\mathbb{Q}) / \mathbb{Q}}^{p}\right)(\log D)
$$

where for $U$ Zariski open, def $/ \mathbb{Q}$ in $S$, write $U=X-D, D$ divisor with normal crossings.

(d) The map

$$
H^{q}\left(U, \Omega_{\mathcal{S}(X)(\mathbb{Q}) / \mathbb{Q}}^{p}(\log D)\right) \stackrel{\mathcal{L}_{\theta_{1}}}{\longrightarrow} H^{q+1}\left(U, \Omega_{\mathcal{S}(X)(\mathbb{Q}) / \mathbb{Q}}^{p}(\log D)\right)
$$

is zero.

Proof of (d): Since $\left.\left.\mathcal{L}_{\theta_{1}}=(\rfloor \theta_{1}\right) \circ d-d \circ(\rfloor \theta_{1}\right)$, we just need that

$$
H^{q}\left(U, \Omega_{\mathcal{S}(X)(\mathbb{Q}) / \mathbb{Q}}^{p}(\log D)\right) \stackrel{d}{\longrightarrow} H^{q}\left(0, \Omega_{\mathcal{S}(X)(\mathbb{Q}) / \mathbb{Q}}^{p+1}(\log D)\right)
$$

is zero. But this follows by base change from showing

$$
H^{q}\left(U, \Omega_{\mathcal{S}(X)(\mathbb{C}) / \mathbb{C}}^{p}(\log D)\right) \stackrel{d}{\longrightarrow} H^{1}\left(U, \Omega_{\mathcal{S}(X)(\mathbb{C}) / \mathbb{C}}^{p+1}(\log D)\right)
$$

is zero. This follows from degeneracy of the Hodge-De Rham spectral sequence for logarithmic differentials.

To conclude this section we shall give for later use a technical result which, however, has interesting geometric content. For this we consider the diagram

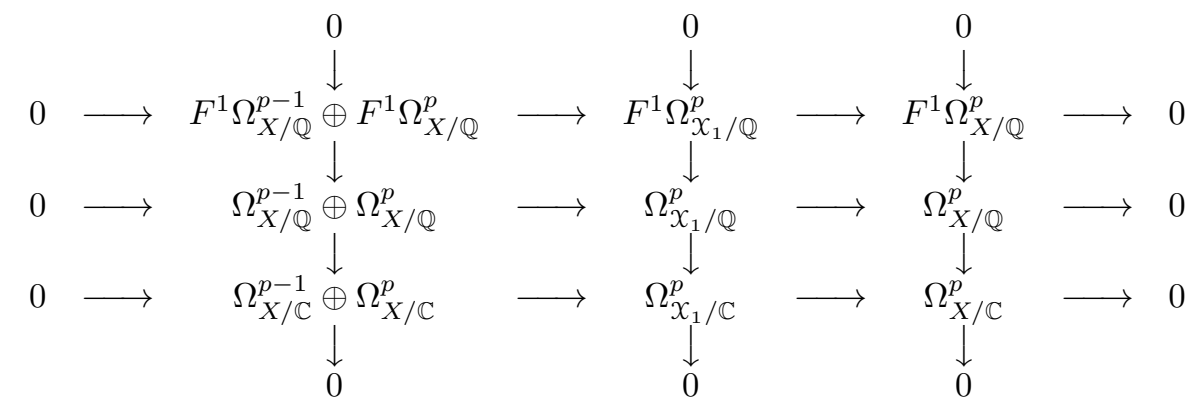

A piece of the cohomology diagram is, using proposition (2(i).11), given by

$$
\begin{aligned}
& 0 \\
& \downarrow \\
& F^{1} H^{q}\left(\Omega_{X / \mathbb{Q}}^{p}\right) \\
& H^{q}\left(\Omega_{X_{1} / \mathbb{Q}}^{p}\right) \quad \longrightarrow \quad H^{q}\left(\Omega_{X / \mathbb{Q}}^{p}\right) \stackrel{\theta,\rfloor}{\longrightarrow} H^{q+1}\left(\Omega_{X / \mathbb{Q}}^{p-1}\right) \\
& H^{q}\left(\Omega_{X / \mathbb{C}}^{p}\right) \stackrel{\left.\theta_{1}\right\rfloor}{\longrightarrow} H^{q+1}\left(\Omega_{X / \mathbb{C}}^{p-1}\right) .
\end{aligned}
$$


The replacement of $H^{q}\left(F^{1} \Omega_{X / \mathbb{Q}}^{p}\right)$ by $F^{1} H^{q}\left(\Omega_{X / \mathbb{Q}}^{p}\right)$ will be explained below.

(2(i).13) Proposition: Let $\eta \in H^{q}\left(\Omega_{X / \mathbb{Q}}^{p}\right)$ have image denoted by $\eta_{\mathbb{C}} \in H^{q}\left(\Omega_{X / \mathbb{C}}^{p}\right)$. Suppose that

$$
\left.\theta_{1}\right\rfloor \eta_{\mathbb{C}}=0
$$

in $H^{q+1}\left(\Omega_{X / \mathbb{C}}^{p-1}\right)$. Then we can find $\eta^{\prime} \in F^{1} H^{q}\left(\Omega_{X / \mathbb{C}}^{p}\right)$ such that

$$
\left.\theta_{1}\right\rfloor\left(\eta+\eta^{\prime}\right)=0
$$

in $H^{q+1}\left(\Omega_{X / \mathbb{C}}^{p-1}\right)$.

(2(i).14) Corollary: Under the assumptions of the proposition, we may modify $\eta$ by $\eta^{\prime} \in F^{1} H^{q}\left(\Omega_{X / \mathbb{C}}^{p}\right)$ so that $\eta+\eta^{\prime}$ lifts to $H^{q}\left(\Omega_{x_{1} / \mathbb{Q}}^{p}\right)$.

Geometrically, $\eta_{\mathbb{C}} \in H^{q}\left(\Omega_{X / \mathbb{C}}^{p}\right)=H^{p, q}(X) \subset H^{p+q}(X, \mathbb{C})$. The assumption in the proposition is that, to $1^{\text {st }}$ order, $\eta_{\mathbb{C}}$ remains of Hodge type $(p, q)$ when we identify all the $H^{p+q}\left(X_{t}, \mathbb{C}\right)$ with $H^{p, q}(X, \mathbb{C})$ for $\left|t-t_{0}\right|<\epsilon$ (Gauss-Manin connection). This implies that $\eta_{\mathbb{C}}$ lifts to $H^{q}\left(\Omega_{X_{1} / \mathbb{C}}^{p}\right)$. What the proposition states is that we may modify the absolute class $\eta \in H^{q}\left(\Omega_{X / \mathbb{Q}}^{p}\right)$ by $\eta^{\prime}$ keeping the same $\left(\eta+\eta^{\prime}\right)_{\mathbb{C}}=\eta_{\mathbb{C}}$ so that $\eta+\eta^{\prime}$ lifts to $H^{q}\left(\Omega_{x_{1} / \mathbb{Q}}^{p}\right)$.

Proof of (2(i).13): We first recall the relative deRham formulation of the degeneracy at $E_{2}$ of the Leray spectral sequence of a proper smooth mapping

$$
W \stackrel{f}{\longrightarrow} S
$$

of quisi-projective varieties, where for our application we may take $S$ to be affine. Now $\Omega_{W / \mathbb{C}}^{\bullet}$ is filtered by

$$
F^{m} \Omega_{W / \mathbb{C}}^{\bullet}=\text { image }\left\{f^{*}\left(\Omega_{S / \mathbb{C}}^{m}\right) \otimes \Omega_{W / \mathbb{C}}^{\bullet-m} \rightarrow \Omega_{W / \mathbb{C}}^{\bullet}\right\}
$$

with

$$
G r^{m} \Omega_{W / \mathbb{C}}^{\bullet} \cong \Omega_{S / \mathbb{C}}^{m} \otimes \Omega_{W / S}^{\bullet-m}
$$

(we drop the $f^{*}$ ). Since $S$ is assumed to be affine the Leray spectral sequence has

$$
E_{1}^{p, q}=H^{0}\left(\Omega_{S / \mathbb{C}}^{p}\right) \otimes \mathbb{H}^{q}\left(\Omega_{W / S}^{\bullet}\right)
$$

and

$$
d_{1}=\nabla_{W / S}
$$

is the Gauss-Manin connection. By the theorem of Blanchard-Deligne this spectral degenerates at $E_{2}$. The cohomology group

$$
H^{q}(W) \cong \mathbb{H}^{q}\left(\Omega_{W / \mathbb{C}}^{\bullet}\right)
$$

has a natural filtration with

$$
G r^{p} H^{q}(W) \cong\left(H^{0}\left(\Omega_{S / \mathbb{C}}^{p}\right) \otimes \mathbb{H}^{p+q}\left(\Omega_{W / S}^{\bullet}\right)\right)_{\nabla_{W / S}}
$$

where ()$_{\nabla_{W / S}}$ denotes the cohomology of the sequence

$$
\cdots \rightarrow H^{0}\left(\Omega_{S / \mathbb{C}}^{p}\right) \otimes \mathbb{H}^{q}\left(\Omega_{W / S}^{\bullet}\right) \stackrel{\nabla_{W / S}}{\longrightarrow} H^{0}\left(\Omega_{S / \mathbb{C}}^{p+1}\right) \otimes \mathbb{H}^{q}\left(\Omega_{W / S}^{\bullet}\right) \rightarrow \cdots
$$


Suppose now that we have

$$
\mathcal{W} \rightarrow S \times T
$$

which we think of as a family of families

$$
W_{t} \rightarrow S, \quad t \in T \text { and } W_{0}=W
$$

to each of which the above considerations apply. We then have

$$
\cdots \rightarrow \Omega_{T / \mathbb{C}}^{p} \otimes \mathbb{H}^{q}\left(\Omega_{\mathcal{W} / T}^{\bullet}\right) \stackrel{\nabla_{\mathcal{W} / T}}{\longrightarrow} \Omega_{T / \mathbb{C}}^{p+1} \otimes \mathbb{H}^{q}\left(\Omega_{\mathcal{W} / T}^{\bullet}\right) \rightarrow \cdots
$$

The issue we want to consider is whether $\nabla_{\mathcal{W} / T}$ induces non-trivial maps

$$
\begin{aligned}
& \operatorname{ker}\left(G r^{p} H^{q}(W)\right.\left.\stackrel{\nabla_{\mathcal{W} / T}}{\longrightarrow} \Omega_{T / \mathbb{C}}^{q} \otimes G r^{p} H^{q}(W)\right) \\
& \longrightarrow \operatorname{coker}\left(G r^{p+1} H^{q}(W) \stackrel{\nabla_{\mathcal{W} / T}}{\longrightarrow} \Omega_{T / \mathbb{C}}^{q} \otimes G r^{p+1} H^{q}(W)\right)
\end{aligned}
$$

We may rewrite this as

$$
\begin{aligned}
\operatorname{ker}\left\{\left(H^{0}\left(\Omega_{S / \mathbb{C}}^{p}\right) \otimes \mathbb{H}^{q-p}\left(\Omega_{W / S}^{\bullet}\right)\right)_{\nabla_{W / S}}\right. \\
\left.\longrightarrow \Omega_{T / \mathbb{C}}^{1} \otimes\left(H^{0}\left(\Omega_{S / \mathbb{C}}^{p} \otimes \mathbb{H}^{q-p}\left(\Omega_{W / S}^{\bullet}\right)\right)\right)\right\}_{\nabla_{W / S}} \\
\longrightarrow \text { coker }\{\text { similar groups }\} .
\end{aligned}
$$

Now, and this is the point, assuming that $T$ is contractible

$$
\mathcal{W} \longrightarrow S \times T
$$

has its own Leray spectral sequence which degenerates at $E_{2}$, and we claim that the maps in (2(i).15) are part of $d_{2}$ for this spectral sequence. First, denoting by $X$ a typical fibre of $W \rightarrow S$ we have that

$$
\mathbb{H}^{q}\left(\Omega_{\mathcal{W} / S \times T}^{\bullet}\right) \cong \mathbb{H}^{q}\left(\Omega_{W / S}^{\bullet}\right)
$$

localizes over $s_{0} \in S$ to $H^{q}(X)$. Now

$$
\left(H^{0}\left(\Omega_{S \times T / \mathbb{C}}^{p}\right) \otimes H^{q-p}(X)\right)_{\nabla_{\mathcal{W} / S \times T}} \stackrel{d_{2}}{\longrightarrow}\left(H^{0}\left(\Omega_{S \times T / \mathbb{C}}^{p+2}\right) \otimes H^{q-p+1}(X)\right)_{\nabla_{\mathcal{W} / S \times T}} .
$$

The LHS contains

$$
\operatorname{ker}\left\{\left(H^{0}\left(\Omega_{S / \mathbb{C}}^{p}\right) \otimes H^{q-p}(X)\right)_{\nabla_{\mathcal{W} / S}} \stackrel{\nabla_{\mathcal{W} / T}}{\longrightarrow} \Omega_{T / \mathbb{C}}^{1} \otimes H^{0}\left(\Omega_{S / \mathbb{C}}^{p}\right) \otimes H^{q-p}(X)\right\},
$$

the RHS contains

$$
\frac{\Omega_{T / \mathbb{C}}^{1} \otimes\left(H^{0}\left(\Omega_{S / \mathbb{C}}^{p+2}\right) \otimes H^{q-p+1}(X)\right)_{\nabla_{W / S}}}{\nabla_{\mathcal{W} / T}\left(H^{0}\left(\Omega_{S / \mathbb{C}}^{p+1}\right) \otimes H^{q-p+1}(X)\right)_{\nabla_{W / S}}}
$$

and the $d_{2}$ mapping contains (2(i).15).

Finally, we apply these considerations to the situation where

$$
\mathcal{W} \rightarrow T \times S
$$


is given by

$$
\begin{array}{lll}
\mathcal{W}_{t} & =\mathcal{S}\left(X_{t}\right) \\
\downarrow & & \downarrow \\
S & = & \stackrel{S}{S}
\end{array}, \quad t \in T(k)
$$

and

$$
\begin{array}{ccc}
\mathcal{W} \mid T \times\left\{s_{0}\right\} & = & x \\
\downarrow & & \downarrow \\
T & = & T
\end{array}
$$

to conclude (2(i).13).

(ii) First order obstructions for lifting Chow groups

Basic assumption: We are working modulo torsion, and for simplicity of notation we use

$$
C H^{p}(X)
$$

to denote the usual Chow group modulo torsion. By basic results of Bloch-Quillen and Soulé we have

$$
C H^{p}(X) \cong H^{p}\left(\mathcal{K}_{p}^{M}\left(\mathcal{O}_{X}\right)\right)
$$

where $\mathcal{K}_{p}^{M}\left(\mathcal{O}_{X}\right)$ is the sheaf (in the Zariski topology) given by the $p^{\text {th }}$ Milnor $K$-groups constructed from the local rings $\mathcal{O}_{X, x}$.

The basic idea is to make the identification (2(ii).1) and then calculate the obstruction to lifting a class in $H^{p}\left(\mathcal{K}_{p}^{M}\left(\mathcal{O}_{X}\right)\right)$ over the successive infinitesimal neighborhoods $X_{k}$ of $X$ in $X$. For this the basic sequence following Van der Kallen and Bloch is

$$
0 \longrightarrow \Omega_{X / \mathbb{Q}}^{p-1} \longrightarrow \mathcal{K}_{p}^{M}\left(\mathcal{O}_{X_{1}}\right) \longrightarrow \mathcal{K}_{p}^{M}\left(\mathcal{O}_{X}\right) \longrightarrow 0 \text {. }
$$

The right hand map is given by restriction of Steinberg symbols

$$
\left\{f_{1}, \ldots, f_{p}\right\} \rightarrow\left\{\left.f_{1}\right|_{X}, \ldots,\left.f_{p}\right|_{X}\right\}, \quad f_{i} \in \mathcal{O}_{X_{1}, x}^{*}
$$

and the left hand map is induced by

$$
f \frac{d g_{1}}{g_{1}} \wedge \cdots \wedge \frac{d g_{p-1}}{g_{p-1}} \rightarrow\left\{1+t \tilde{f}, \tilde{g}_{1}, \ldots \tilde{g}_{p}\right\}, \quad t^{2}=0
$$

where $f \in \mathcal{O}_{X, x}, g_{i} \in \mathcal{O}_{X, x}^{*}$ and $\tilde{f}, \tilde{g}_{1}, \ldots, \tilde{g}_{p}$ denote lifts of these functions to $\mathcal{O}_{x_{1, x}}$. We shall prove later that this mapping is well-defined and that (2(ii).2) is exact. Also, it will simplify the notation to write (2(ii).3) as

$$
f \frac{d g_{1}}{g_{1}} \wedge \cdots \wedge \frac{d g_{p-1}}{g_{p-1}} \rightarrow\left\{1+t f, g_{1}, \ldots g_{p-1}\right\}
$$

with the extensions being understood.

To explain the basic observation behind this work, we recall the arithmetic cycle class mapping

induced by

$$
H^{p}\left(\mathcal{K}_{p}^{M}\left(\mathcal{O}_{X}\right)\right) \stackrel{\eta}{\longrightarrow} H^{p}\left(\Omega_{X / \mathbb{Q}}^{p}\right)
$$

$$
\left\{f_{1}, \ldots, f_{p}\right\} \rightarrow \frac{d f_{1}}{f_{1}} \wedge \cdots \wedge \frac{d f_{p}}{f_{p}}, \quad d=d_{X / \mathbb{Q}}
$$


(we omit the usual $(2 \pi \sqrt{-1})^{-p}$ factor, as it will play no role in this paper and would only serve as a notational distraction).

(2(ii).5) Proposition: The coboundary map $\delta$ in the exact cohomology sequence of (2(ii).2) is given by

$$
\left.\delta(\xi)=\theta_{1}\right\rfloor \eta(\xi), \quad \xi \in H^{p}\left(\mathcal{K}_{p}^{M}\left(\mathcal{O}_{X}\right)\right) .
$$

Since $\delta(\xi)$ is the $1^{\text {st }}$ order obstruction to lifting $\xi$ to $\xi_{1} \in H^{p}\left(\mathcal{K}_{p}^{M}\left(\mathcal{O}_{X_{1}}\right)\right)$, we have the to us remarkable conclusion:

(2(ii).5) The obstruction to lifting $\xi$ to $\xi_{1}$ depends only on the arithmetic cycle class $\eta(\xi)$.

In our paper [G-G1] we have proposed a set of Hodge-theoretic invariants $\varphi_{0}(Z)$, $\ldots, \varphi_{2 p-2}(Z)$ of an algebraic cycle $Z \in Z^{p}(X)$ which, assuming a conjecture of Bloch-Beilinson, would be a complete set of invariants of the rational equivalence class $[Z] \in C H^{p}(X)$ modulo torsion. The invariants $\varphi_{0}(Z), \varphi_{1}(Z), \varphi_{3}(Z), \ldots$, $\varphi_{2 p-3}(Z)$ determine the arithmetic cycle class $\eta(Z)$, so at first glance it appears that the obstruction to lifting $\xi=[Z]$ only "sees" slightly more than one half of the invariants of $\xi$. The actual situation is somewhat subtle and will be discussed later.

For the proof of (2(ii).5) we consider the diagram

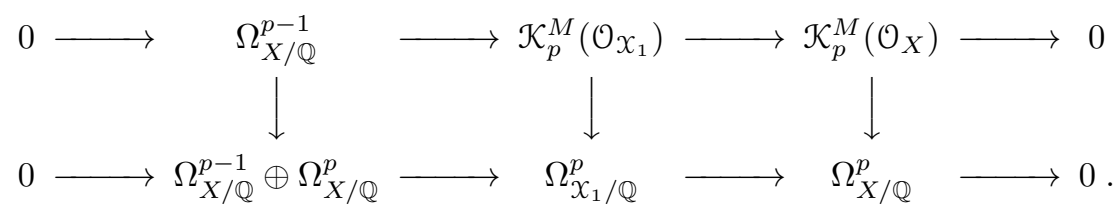

Here, the bottom row is the exact sheaf sequence in proposition (2(i).11), the first map being

$$
\varphi \oplus \omega \mapsto d t \wedge \varphi+t \omega .
$$

The two right hand vertical arrows are $\wedge^{p} d \log$ as in (2(ii).4). The left hand vertical arrow is

$$
\varphi \mapsto \varphi \oplus d \varphi
$$

The commutativity of (2(ii).7) follows from (2(ii).8) and

$$
\wedge^{p} d \log \left\{1+t f, g_{1}, \ldots g_{p-2}\right\}=d t \wedge f \frac{d g_{1}}{g_{1}} \wedge \cdots \wedge \frac{d g_{p-1}}{g_{p-1}}+t d f \wedge \frac{d g_{1}}{g_{1}} \wedge \cdots \wedge \frac{d g_{p-1}}{g_{p-1}} .
$$

Passing to cohomology we have

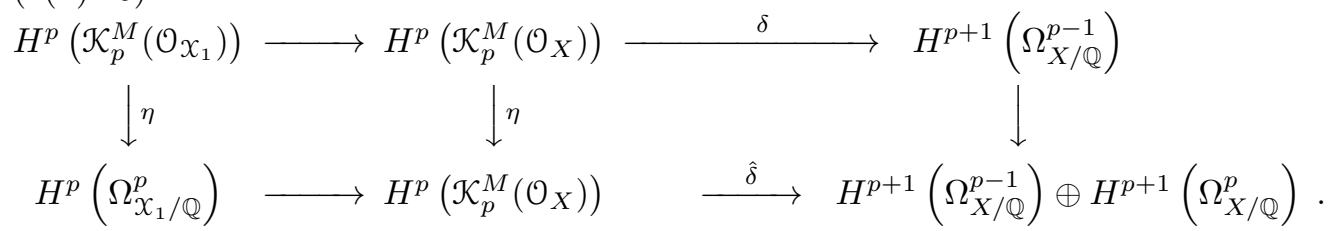

Here, the right hand vertical arrow is, by (2(ii).10)

$$
\varphi \mapsto \varphi \oplus d \varphi, \quad \varphi \in H^{p+1}\left(\Omega_{X / \mathbb{Q}}^{p-1}\right) .
$$


Since by proposition (2(i).11)

$$
\begin{aligned}
\hat{\delta} & \left.=\theta_{1}\right\rfloor \oplus \mathcal{L}_{\theta_{1}} \\
& =\theta_{1}
\end{aligned}
$$

where $\mathcal{L}_{\theta_{1}}=0$ on cohomology, we have

$$
\delta(\xi)=\hat{\delta} \eta(\xi) .
$$

This proves the proposition.

Remark: It is to us quite interesting that the $\Omega_{X / \mathbb{Q}}^{p}$ summand in the bottom row of (2(ii).7), which plays no role in the obstruction to lifting a class in $H^{p}\left(\Omega_{X / \mathbb{Q}}^{p}\right)$ to $H^{p}\left(\Omega_{x_{1} / \mathbb{Q}}^{p}\right)$, is absent from the top row in (2(ii).11). We may say that the geometric question of lifting a class in $H^{p}\left(\mathcal{K}_{p}^{M}\left(\mathcal{O}_{X}\right)\right)$ to $H^{p}\left(\mathcal{K}_{p}^{m}\left(\mathcal{O}_{x_{1}}\right)\right)$ does not see irrelevant information.

We will now give the geometric interpretation of the obstruction in proposition (2(ii).5) and of the conclusion (2(ii).5) in the first two cases $p=1, p=2$; the interpretation in the case $p=2$ will extend to the cases $p \geqq 2$.

$p=1$. In this case - which is classical — the sequence (2(ii).2) reduces to

$$
0 \rightarrow \mathcal{O}_{X} \rightarrow \mathcal{O}_{X_{1}}^{*} \rightarrow \mathcal{O}_{X}^{*} \rightarrow 0
$$

with the first map being

$$
f \rightarrow 1+t f, \quad t^{2}=0
$$

The basic obstruction diagram (2(ii).10) is

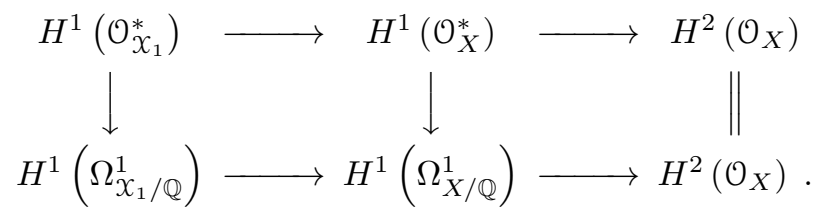

To interpret $\left.\theta_{1}\right\rfloor \eta(\xi)$ where $\xi=H^{1}\left(\mathcal{O}_{X}^{*}\right)$, we have

where the dotted arrow has the interpretation

$$
\eta_{\mathbb{C}}(\xi) \text { is the fundamental class } \psi_{0}(\xi) \in H^{1,1}(X) .
$$

Moreover,

$\left.\left.\theta_{1}\right\rfloor \eta(\xi)=\theta_{1}\right\rfloor \eta_{\mathbb{C}}(\xi)$ is the $1^{\text {st }}$ order deviation of $\psi_{0}(\xi)$ from remaining of Hodge type $(1,1)$ in the family $\left\{X_{t}\right\}_{t \in B}$. 
Geometrically, to lift $\xi \in \operatorname{Pic}(X)$ to $\operatorname{Pic}(X)$, the Chern class of $\xi$ must remain in $H g^{1}\left(X_{t}\right)$ for all $t$, and once this happens $\xi$ lifts since the family $\operatorname{Pic}^{0}\left(X_{t}\right)$ is unobstructed. The above gives the $1^{\text {st }}$ order calculation that this is the case. Nothing new happens here.

$p=2$. In this case the obstruction diagram (2(ii).10) together with the exact cohomology sequence of

$$
0 \rightarrow \Omega_{\mathbb{C} / \mathbb{Q}}^{1} \otimes \mathcal{O}_{X} \rightarrow \Omega_{X / \mathbb{Q}}^{1} \rightarrow \Omega_{X / \mathbb{C}}^{1} \rightarrow 0
$$

combine to give a diagram $\left(\mathcal{K}_{2}^{M}=\mathcal{K}_{2}\right.$ in this case $)$

Here, $\mathcal{O}_{1}(\xi)$ is what we shall call the primary $1^{\text {st }}$ order obstruction to lifting $\xi \in$ $H^{2}\left(\mathcal{K}_{2}\left(\mathcal{O}_{X}\right)\right)$ to $H^{2}\left(\mathcal{K}_{2}\left(\mathcal{O}_{X_{1}}\right)\right)$. From the discussion above we may infer the interpretation

(2(ii).12) The primary $1^{\text {st }}$ order obstruction is given by

$$
\left.\mathcal{O}_{1}(\xi)=\theta_{1}\right\rfloor \psi_{0}(\xi) ;
$$

it represents the $1^{\text {st }}$ order deviation of the fundamental class $\psi_{0}(\xi) \in$ $H^{2,2}(X)$ from remaining of Hodge type $(2,2)$ in the family $\left\{X_{t}\right\}_{t \in B}$.

Proof: Let $\eta_{\mathbb{C}}: H^{2}\left(\mathcal{K}_{2}\left(\mathcal{O}_{X}\right)\right) \rightarrow H^{2}\left(\Omega_{X / \mathbb{C}}^{2}\right)$ be the mapping induced on cohomology by

$$
\left\{f_{1}, f_{2}\right\} \rightarrow d_{\mathbb{C}} \log f_{1} \wedge d_{\mathbb{C}} \log f_{2}
$$

where $d_{\mathbb{C}}=d_{X / \mathbb{C}}$. It is well known that under the isomorphism

$$
H^{2}\left(\Omega_{X / \mathbb{C}}^{2}\right) \cong H^{2,2}(X)
$$

we have (up to the factor $(2 \pi \sqrt{-1})^{-2}$ )

$$
\eta_{\mathbb{C}}(\xi)=\psi_{0}(\xi)
$$

corresponds to the fundamental class of $\xi$. Since

$$
\left.\mathcal{O}_{1}(\xi)=\theta_{1}\right\rfloor \eta_{\mathbb{C}}(\xi)
$$

the result (2(ii).12) follows. 
In (2(ii).11) the dotted arrow means that $\mathcal{O}_{2}(\xi)$ is defined only if $\mathcal{O}_{1}(\xi)=0$. Suppose this is the case. We shall refer to $\mathcal{O}_{2}(\xi)$ as the secondary $1^{\text {st }}$ order obstruction to lifting $\xi=H^{2}\left(\mathcal{K}_{2}\left(\mathcal{O}_{X}\right)\right)$ to $H^{2}\left(\mathcal{K}_{2}\left(\mathcal{O}_{X_{1}}\right)\right)$, and we shall now give its geometric interpretation.

For this we need a short discussion recalling some of the material from [G-G1]. Suppose that $\xi=[Z] \in C H^{p}(X(k))$ where both $X$ and $Z$ are defined over field $k$. Taking $k$-spreads we have first the spread

$$
\mathcal{S}(X) \stackrel{\tilde{\omega}}{\longrightarrow} S
$$

of $X$ and then the spread

$$
\mathcal{S}(Z) \in Z^{p}(\mathcal{S}(X)(\mathbb{Q}))
$$

of $Z$. Both $\mathcal{S}(X)$ and $\mathcal{S}(Z)$ are only defined up to ambiguities as discussed in (loc. cit). The arithmetic cycle class

$$
\eta(\mathcal{S}(Z)) \in H^{p}\left(\Omega_{\mathcal{S}(X)(\mathbb{Q}) / \mathbb{Q}}^{p}\right)
$$

By base change

$$
H^{p}\left(\Omega_{\mathcal{S}(X)(\mathbb{Q}) / \mathbb{Q}}^{p}\right) \otimes \mathbb{C}=H^{p}\left(\Omega_{\mathcal{S}(X) / \mathbb{C}}^{p}\right) \subset H^{2 p}(\mathcal{S}(X), \mathbb{C})
$$

and under this inclusion

$$
\eta(\mathcal{S}(Z)) \in H g^{p}(\mathcal{S}(X))
$$

is the fundamental class of $\mathcal{S}(Z)$. We shall denote by

$$
\psi_{0}(\mathcal{S}(Z)) \in H^{2 p}(\mathcal{S}(X), \mathbb{C}) / \text { ambiguities }
$$

the image of $\eta(\mathcal{S}(Z))$ when we factor out the ambiguities in $H^{2 p}(\mathcal{S}(X), \mathbb{C})$ induced by the ambiguities in (2(ii).13) and (2(ii).14). From (loc. cit) we have

$$
\psi_{0}(\mathcal{S}(Z)) \text { may be identified with } \eta(\xi) \text {. }
$$

This has the following precise meaning. We consider the Leray spectral sequence of (2(ii).13) for the sheaf $\Omega_{\mathcal{S}(X) / \mathbb{C}}^{r}$ and localized over the generic point $s_{0} \in S$. This spectral sequence degenerates at $E_{2}$ (cf. [E-P]), and making the identification

$$
\Omega_{S / \mathbb{C}, s_{0}}^{1}=\Omega_{k / \mathbb{Q}}^{1} \otimes \mathbb{C} \subset \Omega_{\mathbb{C} / \mathbb{Q}}^{1}
$$

we have (loc. cit)

$$
E_{2}^{p, q}=\left(\Omega_{\mathbb{C} / \mathbb{Q}}^{q} \otimes H^{p+q}\left(\Omega_{X / \mathbb{C}}^{r}\right)\right)_{\nabla_{a}} .
$$

Here the right hand side is the cohomology of the complex

$$
\Omega_{\mathbb{C} / \mathbb{Q}}^{q-1} \otimes H^{p+q}\left(\Omega_{X / \mathbb{C}}^{+1}\right) \stackrel{\nabla_{a}}{\longrightarrow} \Omega_{\mathbb{C} / \mathbb{Q}}^{q} \otimes H^{+q}\left(\Omega_{X / \mathbb{C}}\right) \stackrel{\nabla_{a}}{\longrightarrow} \Omega_{\mathbb{C} / \mathbb{Q}}^{q+1} \otimes H^{p+q}\left(\Omega_{X / \mathbb{C}}^{-1}\right)
$$

where $\nabla_{a}$ is the arithmetic Gauss-Manin connection. This spectral sequence abuts to

$$
H^{p+q}\left(\Omega_{\mathcal{S}(X) / \mathbb{C}}^{r}\right) \text { localized at } s_{0} \cong H^{p+q}\left(\Omega_{X / \mathbb{Q}}^{r}\right)
$$

where the isomorphism results from (2(ii).16). Taking $q=0$ and $r=p, \psi_{0}(\mathcal{S}(Z))$ belongs to the LHS in (2(ii).18), $\eta(\xi)$ belongs to the RHS and under this identification

$$
\psi_{0}(\mathcal{S}(Z)) \rightarrow \eta(\xi)
$$


Finally, from [G-G1]

$$
\left\{H^{p}\left(\Omega_{\mathcal{S}(X) / \mathbb{C}}^{p}\right) \text { localized at } s_{0}\right\} \cong H^{p}\left(\Omega_{\mathcal{S}(X) / \mathbb{C}}^{p}\right) / \text { ambiguities. }
$$

Combining (2(ii).18) and (2(ii).20) we see that the map (2(ii).19) is injective, and this is the meaning of (2(ii).15).

After these preliminaries we may finally give the geometric interpretation of the secondary $1^{\text {st }}$ order obstruction. Namely, if $\xi \in H^{2}\left(\mathcal{K}_{2}\left(\mathcal{O}_{X}\right)\right)$ deforms to $\xi_{t} \in$ $H^{2}\left(\mathcal{K}_{2}\left(\mathcal{O}_{X_{t}}\right)\right)$ then we will have $\xi_{t}=\left[Z_{t}\right]$ for a family of cycles $Z_{t} \in Z^{2}\left(X_{t}\right)$. Assuming everything is defined over $k$, we will have the family of spreads

$$
\left\{\begin{array}{l}
\mathcal{S}\left(X_{t}\right) \longrightarrow S, \\
\mathcal{S}\left(Z_{t}\right) \in Z^{2}\left(X\left(X_{t}\right)(\mathbb{Q})\right) .
\end{array} \quad t \in B(k)\right.
$$

In particular,

$$
\psi_{0}(\mathcal{S}(Z)) \text { remains of Hodge type }(2,2) \text { in the family (2(ii).21). }
$$

The vanishing of the primary $1^{\text {st }}$ order obstruction $\mathcal{O}_{1}(\xi)$ is the $1^{\text {st }}$ order implication of this in the top piece of the Leray filtration on $H^{4}(\mathcal{S}(X), \mathbb{C})$ mod ambiguities. Assuming $\mathcal{O}_{1}(\xi)=0$, the vanishing of $\mathcal{O}_{2}(\xi)$ is well-defined and geometrically gives the condition that in (2(ii).22) the second piece in the Leray filtration of $H^{4}(\mathcal{S}(X), \mathbb{C}) \bmod$ ambiguities be true to $1^{\text {st }}$ order.

This interpretation extends in the evident way for all $p$. We may summarize the above discussion as follows:

(2(ii).23) The $1^{\text {st }}$ order obstruction to lifting a rational equivalence class $[Z] \in$ $C H^{p}(X)$ is expressed by the diagram

There are filtrations on $C H^{p}(X) \cong H^{p}\left(\mathcal{K}_{p}^{M}\left(\mathcal{O}_{X}\right)\right), H^{p}\left(\Omega_{X / \mathbb{Q}}^{p}\right)$ and $H^{p+1}\left(\Omega_{X / \mathbb{Q}}^{p-1}\right)$, and the maps in the above diagram all preserve these filtrations. The filtration on the obstruction space $H^{p+1}\left(\Omega_{X / \mathbb{Q}}^{p-1}\right)$ reflects the geometric condition that, to $1^{\text {st }}$ order, the pieces of the fundamental 
class of the spread $\mathcal{S}(Z)$ in the Leray filtration on $H^{2 p}(\mathcal{S}(X), \mathbb{C})$ mod ambiguities remain of Hodge type $(p, p)$.

Since the filtrations on $C H^{p}(X)$ and $H^{p}\left(\Omega_{X / \mathbb{Q}}^{p}\right)$ have $p$ steps and the filtration on the obstruction space $H^{p+1}\left(\Omega_{X / \mathbb{Q}}^{p-1}\right)$ has only $p-1$ steps, and since the obstruction map preserves the filtrations, we may infer the following

(2(ii).24) Corollary: To $1^{\text {st }}$ order, $F^{p} C H^{p}(X)$ is unobstructed.

For $p=1$ this is the well-known fact that $\operatorname{Pic}^{0}(X)$ moves smoothly with $X$ in a family. For $p \geqq 2$ this appears to be a new observation whose geometric interpretation we will discuss later.

\section{Higher ORDER OBSTRUCTION THEORY}

\section{(i) Higher order Kodaira-Spencer theory}

There are two well-developed systematic higher order Kodaira-Spencer theories $[\mathrm{EV}],[\mathrm{R}]$. The rudimentary theory given here is amenable to the computations we need to make.

Recalling the exact sequence (2(i).1) we give the

Definition: The $m^{\text {th }}$ order Kodaira-Spencer class is

$$
\theta_{m}=\delta(\partial / \partial t) \in H^{1}\left(\Theta_{m-1}\right) .
$$

For $m=1$ we have the usual $1^{\text {st }}$ order Kodaira-Spencer class $\theta_{1} \in H^{1}\left(\Theta_{X}\right)$. The class $\theta_{m}$ depends on the choice of local coordinate $t$ on $B$; however we observe that it's vanishing does not depend on this choice

$$
\theta_{m}=0 \text { if, and only if, } x_{m} \cong X \times \operatorname{Spec}\left(\mathbb{C}[t] / t^{m+1}\right) .
$$

The above definition is convenient for calculations, and the geometric conclusions that will be drawn from these calculations will not depend on the choice of parameter $t$. One may of course give an intrinsic definition, but for our purposes this is not necessary. Remark that a change of parameter

$$
t \rightarrow a t+b t^{2}
$$

induces

$$
\begin{aligned}
& \theta_{1} \longrightarrow\left(\frac{1}{a}\right) \theta_{1} \\
& \theta_{2} \longrightarrow\left(\frac{1}{a}\right)\left[\theta_{2}+\left(\frac{b}{a}\right) \theta_{1}\right],
\end{aligned}
$$

from which the general pattern of how the $\theta_{m}$ transform is clear.

Using the notation (2(i).6) and setting

$$
\tilde{\theta}_{m \alpha \beta}=f_{\alpha \beta m}\left(z_{\beta}\right) \partial / \partial z_{\alpha}
$$

we have

$$
e_{\beta}(\partial / \partial t)-e_{\alpha}(\partial / \partial t)=\tilde{\theta}_{1 \alpha \beta}+t \tilde{\theta}_{2 \alpha \beta}+\cdots .
$$


Thus the Čech representative cocycles for $\theta_{1}, \theta_{2}, \ldots$ are given by

$$
\left\{\begin{aligned}
\theta_{1 \alpha \beta} & =\tilde{\theta}_{1 \alpha \beta} \\
\theta_{2 \alpha \beta} & =\tilde{\theta}_{1 \alpha \beta}+t \tilde{\theta}_{2 \alpha \beta} \\
\vdots &
\end{aligned}\right.
$$

Under a change of $t$ as above

$$
\begin{aligned}
& \tilde{\theta}_{1 \alpha \beta} \longrightarrow\left(\frac{1}{a}\right) \tilde{\theta}_{1 \alpha \beta} \\
& \tilde{\theta}_{2 \alpha \beta} \longrightarrow\left(\frac{1}{a}\right) \tilde{\theta}_{2 \alpha \beta}+\left(\frac{b}{a^{2}}\right) \tilde{\theta}_{1 \alpha \beta} .
\end{aligned}
$$

Since $\Theta_{X / B}$ is a sheaf of Lie algebras and since vertical vector fields for $X \rightarrow B$ vanish on the function $t$, the $\Theta_{m}$ are sheaves of Lie algebras. Moreover, there is an exact sequence

$$
0 \longrightarrow \Theta_{X} \stackrel{t^{m}}{\longrightarrow} \Theta_{m} \longrightarrow \Theta_{m-1} \longrightarrow 0, \quad m \geqq 1 .
$$

We claim that:

(3(i).4) The extension class of (3(i).3) is given by an element

$$
e_{m} \in H^{1}\left(\operatorname{Der}_{\mathbb{C}}\left(\Theta_{m-1}, \Theta_{X}\right)\right),
$$

where $\operatorname{Der}_{\mathbb{C}}\left(\Theta_{m-1}, \Theta_{X}\right)$ are the $\mathbb{C}$-linear Lie algebra homomorphisms.

Proof: The cases $m=1,2$ will illustrate the general pattern. Using again the notation (2(i).6) and setting $j_{\alpha \beta}\left(z_{\beta}\right)=\partial_{z_{\beta}} f_{\alpha \beta}\left(z_{\beta}\right)$, we have in $U_{\alpha \beta}$

$$
\begin{aligned}
d z_{\alpha}=j_{\alpha \beta}\left(z_{\beta}\right)\{ & 1+t \partial_{z_{\beta}} f_{\alpha \beta 1}\left(z_{\beta}\right) j_{\alpha \beta}^{-1}\left(z_{\beta}\right) \\
& \left.+\frac{t^{2}}{2} \partial_{z_{\beta}} f_{\alpha \beta 2}\left(z_{\beta}\right) j_{\alpha \beta}^{-1}\left(z_{\beta}\right)+\cdots\right\} d z_{\beta} \bmod d t .
\end{aligned}
$$

If we take the difference of the box coordinate liftings of $\partial / \partial z_{\alpha}$ in $U_{\beta}$ and $U_{\alpha}$ we have

$$
\begin{aligned}
e_{\beta}\left(\partial / \partial z_{\alpha}\right)-e_{\alpha}\left(\partial / \partial z_{\alpha}\right) & =-\left(t \partial_{z_{\alpha}} f_{\alpha \beta 1}\left(z_{\beta}\right)+\frac{t^{2}}{2} \partial_{z_{\alpha}} f_{\alpha \beta 2}\left(z_{\beta}\right)+\cdots\right) \partial / \partial z_{\alpha} \\
& =\left[\left(t f_{\alpha \beta 1}\left(z_{\beta}\right)+\frac{t^{2}}{2} f_{\alpha \beta 2}\left(z_{\beta}\right)+\cdots\right) \partial / \partial z_{\alpha}, \partial / \partial z_{\alpha}\right] .
\end{aligned}
$$

It follows that for $v, w$ sections of $\Theta_{X}$ over $U_{\alpha \beta}$

$$
\left\{\begin{aligned}
e_{1 \alpha \beta}(v) & =\left[\tilde{\theta}_{1 \alpha \beta}, v\right] \\
e_{2 \alpha \beta}(v+t w) & =\left[\tilde{\theta}_{2 \alpha \beta}, w\right]+\frac{1}{2}\left[\tilde{\theta}_{1 \alpha \beta}, v\right]
\end{aligned}\right.
$$

using the notation (3(i).1). The fact that the extension classes are in

$$
\operatorname{Der}_{\mathbb{C}}\left(\Theta_{m-1}, \Theta_{X}\right)
$$

is a consequence of the Jacobi identity.

Comparing (3(i).4) and (3(i).5) we see that

$$
\theta_{m} \text { is constructed from } \theta_{m-1} \text { and } e_{m} \text {. }
$$


Thus, for $m=1$ we have

$$
0 \longrightarrow \Theta_{X} \longrightarrow \Theta_{1} \longrightarrow \Theta_{X} \longrightarrow 0
$$

and the extension class

$$
e_{1}=\theta_{1}
$$

From

we have

$$
e_{1}\left(\theta_{1}\right)=\left[\theta_{1}, \theta_{1}\right]=0
$$

and by (3(i).4)

$$
\left[\theta_{1}, \theta_{1}\right]=\delta \tilde{\theta}_{2}
$$

In general, we will have

$$
\theta_{2 \alpha \beta}=\tilde{\theta}_{1 \alpha \beta}+t \tilde{\theta}_{2 \alpha \beta}
$$

$$
e_{m}=\theta_{m-1} \in H^{1}\left(\operatorname{Der}_{\mathbb{C}}\left(\Theta_{m-1}, \Theta_{X}\right)\right)
$$

and

leading to

$$
e\left(\theta_{m-1}\right)=\left[\theta_{m-1}, \theta_{m-1}\right]=\delta \tilde{\theta}_{m}
$$

$$
\theta_{m \alpha \beta}=\theta_{m-1 \alpha \beta}+t^{m} \tilde{\theta}_{m \alpha \beta} .
$$

In addition to the sequence (2(i).1) we now consider the sequences analogous to $(2(\mathrm{i}) .3)$

$$
\left\{\begin{array}{lll}
\text { (i) } & 0 \longrightarrow \mathcal{O}_{X} \longrightarrow \mathcal{O}_{X_{m}} \longrightarrow \mathcal{O}_{X_{m-1}} \longrightarrow 0 & m \geqq 1 \\
\text { (ii) })_{1} & 0 \longrightarrow \mathcal{O}_{X} \oplus \Omega_{X / \mathbb{C}}^{1} \longrightarrow \Omega_{X_{m} / \mathbb{C}}^{1} \longrightarrow \Omega_{X_{m-1} / \mathbb{C}}^{1} \longrightarrow 0 & m \geqq 1 \\
\text { (iii) })_{1} & 0 \longrightarrow \mathcal{O}_{X} \oplus \Omega_{X / \mathbb{Q}}^{1} \longrightarrow \Omega_{X_{m} / \mathbb{Q}}^{1} \longrightarrow \Omega_{X_{m-1}}^{1} \longrightarrow \mathbb{Q} & m \geqq 1
\end{array}\right.
$$

(3(i).7) Proposition: The extension classes of (3(i).6) are all induced from $\theta_{m}$. Specifically, there are maps

$$
\left\{\begin{array}{l}
\Theta_{m-1} \longrightarrow \operatorname{Der}_{\mathbb{C}}\left(\mathcal{O}_{x_{m-1}}, \mathcal{O}_{X}\right) \\
\Theta_{m-1} \longrightarrow \operatorname{Der}_{\mathbb{C}}\left(\Omega_{X_{m-1} / k}^{1}, \mathcal{O}_{X} \oplus \Omega_{X / k}^{1}\right) \quad k=\mathbb{C}, \mathbb{Q}
\end{array}\right.
$$

which induce maps

$$
\left\{\begin{array}{l}
H^{1}\left(\Theta_{m-1}\right) \longrightarrow H^{1}\left(\operatorname{Der}_{\mathbb{C}}\left(\mathcal{O}_{X_{m-1}}, \mathcal{O}_{X}\right)\right) \\
H^{1}\left(\Theta_{m-1}\right) \longrightarrow H^{1}\left(\operatorname{Der}_{\mathbb{C}}\left(\Omega_{X_{m-1} / k}^{1}, \mathcal{O}_{X} \oplus \Omega_{X / k}^{1}\right)\right)
\end{array}\right.
$$

under which $\theta_{m}$ goes to corresponding extension classes.

Proof: The inclusion

$$
\Theta_{x / B} \subset \Theta_{x}
$$

and pairing

$$
\Theta_{x} \otimes_{\mathbb{C}} \mathcal{O}_{x} \longrightarrow \mathcal{O}_{x}
$$

induce

$$
\Theta_{m-1} \otimes_{\mathbb{C}} \mathcal{O}_{X_{m-1}} \longrightarrow \mathcal{O}_{X_{m-1}} .
$$

The case $m=1$ of the proposition is given in the proof of proposition (2(i).5). For the case $m=2$ we consider a function $g \in \mathcal{O}_{x_{m-1}, x}$. If $x \in U_{\alpha}$ we write

$$
g=g_{0}\left(z_{\alpha}\right)+t g_{1}\left(z_{\alpha}\right)
$$


and extend $g$ to $e_{\alpha}(g) \in \mathcal{O}_{X_{m}, x}$ using the $\left(z_{\alpha}, t\right)$ box coordinates. If $x \in U_{\beta}$ then we write as in (2(i).6)

$$
z_{\alpha}=f_{\alpha \beta}\left(z_{\beta}\right)+t f_{\alpha \beta 1}\left(z_{\beta}\right)+\frac{t^{2}}{2} f_{\alpha \beta 2}\left(z_{\beta}\right)+\cdots
$$

and look at the $t^{2}$ term in $e_{\beta}(g)-e_{\alpha}(g)$; i.e.,

$$
e_{\beta}(g)-e_{\alpha}(g)=\frac{1}{2} f_{\alpha \beta 2} \partial_{z_{\alpha}} g_{0}+f_{\alpha \beta 1} \partial_{z_{\alpha}} g_{1}
$$

which was to be proved. We will write the RHS of this equation as $\mathcal{L}_{\theta_{2}} g$ where

$$
\left(\mathcal{L}_{\theta_{2}} g\right)_{\alpha \beta}=\frac{1}{2} \tilde{\theta}_{2 \alpha \beta} g_{0}+\tilde{\theta}_{1 \alpha \beta} g_{1}
$$

A similar calculation for

$$
\omega=g_{0}\left(z_{\alpha}\right) d z_{\alpha}+t g_{1}\left(z_{\alpha}\right) d z_{\alpha}
$$

gives

$$
\left.e_{\beta}(\omega)-e_{2}(\omega)=\theta_{2}\right\rfloor \omega \oplus \mathcal{L}_{\theta_{2}} \omega
$$

where the cocycle representatives are

$$
\left\{\begin{array}{l}
\left.\left.\left.\left(\theta_{2}\right\rfloor \omega\right)_{\alpha \beta}=\tilde{\theta}_{2 \alpha \beta}\right\rfloor g_{0}\left(z_{\alpha}\right) d z_{\alpha}+\tilde{\theta}_{1 \alpha \beta}\right\rfloor g_{1}\left(z_{\alpha}\right) d z_{\alpha} \\
\left(\mathcal{L}_{\theta_{2}} \omega\right)_{\alpha \beta}=\mathcal{L}_{\tilde{\theta}_{2 \alpha \beta}}\left(g_{0}\left(z_{\alpha}\right) d z_{\alpha}\right)+\mathcal{L}_{\tilde{\theta}_{1 \alpha \beta}}\left(g_{1}\left(z_{\alpha}\right) d z_{\alpha}\right)
\end{array}\right.
$$

Definition: We define

$$
\mathcal{L}_{\theta_{m}}: H^{q}\left(\Omega_{X_{m-1} / k}^{p}\right) \longrightarrow H^{q+1}\left(\Omega_{X / k}^{p}\right) \quad k=\mathbb{Q}, \mathbb{C}
$$

to be the $\Omega_{X / k}^{p}$-component of the coboundary map in the exact cohomology sequence of

$$
0 \longrightarrow \Omega_{X / k}^{p-1} \oplus \Omega_{X / k}^{p} \longrightarrow \Omega_{X_{m} / k}^{p} \longrightarrow \Omega_{X_{m-1} / k}^{p} \longrightarrow 0, \quad k \geqq 1 .
$$

As in the case $m=0$ (cf. proposition (2(i).8)) we have

(3(i).10) Proposition: The maps (3(i).8) are all zero.

Proof: As in the proof of (2(i).8) and (2(i).11) it will suffice to do the case $k=\mathbb{C}$. The case $m=2$ will illustrate how the general pattern goes. The coboundary maps in the cohomology sequences of

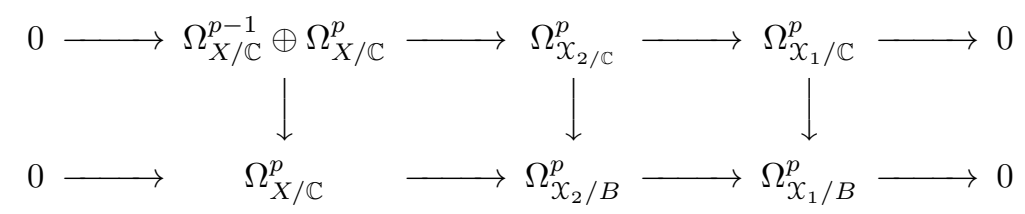

give a commutative diagram

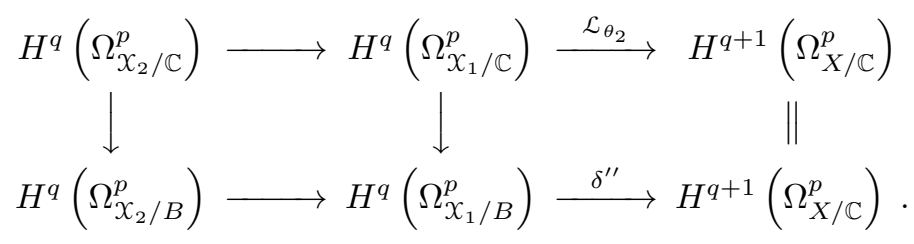


Now $H^{q}\left(\Omega_{x_{m} / B}^{p}\right)$ represents $m$-jets of sections of the vector bundle $R^{q} \Omega_{X / B}^{p} \rightarrow B$ (the fact that this is a locally free sheaf being a consequence of $\operatorname{dim} H^{q}\left(\Omega_{X_{t} / \mathbb{C}}^{p}\right)=$ constant). It follows that $\delta^{\prime \prime}=0$, which implies the result.

The remaining coboundary map in the exact cohomology sequence of (3(i).9) will be denoted by

$$
H^{q}\left(\Omega_{x_{m-1} / k}^{p}\right) \stackrel{\theta_{m}}{\longrightarrow} H^{q+1}\left(\Omega_{X / k}^{p-1}\right) .
$$

It has the following geometric interpretation: An element in $H^{q}\left(\Omega_{x_{m-1} / \mathbb{C}}^{p}\right)$ represents an $(m-1)$-jet of section of $R^{q} \Omega_{x / \mathbb{C}}^{p} \rightarrow B$, and the coboundary

$$
\delta(\varphi)=: \delta^{\prime}(\varphi) \oplus \delta^{\prime \prime}(\varphi) \in H^{q+1}\left(\Omega_{X / \mathbb{C}}^{p-1}\right) \oplus H^{q+1}\left(\Omega_{X / \mathbb{C}}^{p}\right)
$$

represents the obstruction to lifting $\varphi$ to an $m$-jet. By (3(i).8) and (3(i).10)

$$
\delta^{\prime \prime}(\varphi)=\mathcal{L}_{\theta_{m}} \varphi=0 .
$$

We now denote by $\Phi \in\left(R_{\pi}^{p+q} \mathbb{C}\right)_{t_{0}}$ the unique local (in the analytic topology) extension of $\varphi_{0} \in H^{q}\left(\Omega_{X / \mathbb{C}}^{p}\right) \subset H^{p+1}(X, \mathbb{C})$ to a section of $R_{\pi}^{p+q} \mathbb{C}$. By our assumption, to order $m-1$ the section corresponding $\Phi$ of $R_{\pi}^{p+q} \mathbb{C} \otimes \mathcal{O}_{B}$ lies in the sub-bundle

$$
F^{p}\left(R_{\pi}^{p+q} \otimes \mathcal{O}_{B}\right) \subset R_{\pi}^{p+q} \mathbb{C} \otimes \mathcal{O}_{B}
$$

Then

$$
\left.\theta_{m}\right\rfloor \varphi
$$

represents the deviation of $\Phi$ remaining in the sub-bundle (3(i).12) to order $m$. If we write everything out in terms of Cech cocycles as above we see that $\left.\theta_{m}\right\rfloor \varphi$ is expressed by an algebraic contraction operator of $\varphi$ with the $m^{\text {th }}$ order KodairaSpencer class $\theta_{m}$.

We conclude this section with a higher order analogue of proposition (2(i).13). The proof will be given elsewhere.

(3(i).13) Proposition: Let $\eta \in H^{q}\left(\Omega_{x_{m-1} / \mathbb{Q}}^{p}\right)$ have image denoted by $\eta_{\mathbb{C}} \in$ $H^{q}\left(\Omega_{x_{m-1} / \mathbb{C}}^{p}\right)$. Suppose that

$$
\left.\theta_{m}\right\rfloor \eta_{\mathbb{C}}=0
$$

is $H^{q+1}\left(\Omega_{X / \mathbb{C}}^{p-1}\right)$. Then there exists $\eta^{\prime} \in F^{q} H^{q}\left(\Omega_{X_{m-1} / \mathbb{C}}^{p}\right)$ such that

$$
\theta\rfloor\left(\eta+\eta^{\prime}\right)=0
$$

in $H^{q+1}\left(\Omega_{X / \mathbb{Q}}^{p-1}\right)$.

(3(i).14) Corollary: Under the assumptions of the proposition, we may modify $\eta$ by $\eta^{\prime} \in F^{1} H^{q}\left(\Omega_{x_{m-1} / \mathbb{Q}}^{p}\right)$ such that $\eta+\eta^{\prime}$ lifts to $H^{q}\left(\Omega_{x_{m} / \mathbb{Q}}^{p}\right)$.

(ii) Higher order obstructions to lifting Chow groups; applications

This section is essentially an amalgam of sections 2(ii) and 3(i). Referring to (2(i).1) and (2(i).2), the basic sequence for the higher order obstruction theory is

$$
0 \longrightarrow \Omega_{X / \mathbb{Q}}^{p-1} \longrightarrow \mathcal{K}_{p}^{M}\left(\mathcal{O}_{X_{m}}\right) \longrightarrow \mathcal{K}_{p}^{M}\left(\mathcal{O}_{x_{m-1}}\right) \longrightarrow 0
$$


Referring to (2(ii).3), the first map is induced by

$$
f \frac{d g_{1}}{g_{1}} \wedge \cdots \wedge \frac{d g_{p-1}}{g_{p-1}} \longrightarrow\left\{1+t^{m} \tilde{f}, \tilde{g}_{1}, \ldots, \tilde{g}_{p-1}\right\}, \quad t^{m+1}=0 .
$$

The exactness of (3(ii).1) follows from the obvious general case of the following result, for simplicity of notation stated here in the case $p=2$.

In the following proposition and its proof, it is convenient to replace $t$ by $\epsilon$, so that $\epsilon^{m+1}=0$.

(3(ii).3) Proposition: Every element of $\mathcal{K}_{2}\left(\mathcal{O}_{x_{m, x}}\right)$ can be written as a product of elements of the form

$$
\left\{f, g_{0}+g_{1} \epsilon+\cdots+g_{m} \epsilon^{m}\right\} \quad f, g_{i} \in \mathcal{O}_{X, x} .
$$

Proof: We will say that a ring $R$ is spanned by powers if for every $d>0$ every $r \in R$ is a sum of $d^{\text {th }}$ powers

$$
r=p_{1}^{d}+\cdots p_{l}^{d}, \quad p_{i} \in R .
$$

Claim 1: $\mathcal{O}_{X, x}$ is spanned by powers.

Proof: We first note that a homogeneous polynomial $r \in \mathbb{C}\left[x_{0}, \ldots, x_{k}\right]$ of degree $k \cdot d$ is a sum of $d^{\text {th }}$ powers; this is just the fact that for $V$ a vector space the image of the Veronese map

spans $\mathbb{P} \operatorname{Sym}^{d} V$.

Next we see that $\mathcal{O}_{\mathbb{P} l, x}$ is spanned by powers: Given $f \in \mathcal{O}_{\mathbb{P}} l, x$ we write

$$
f=P / Q^{d} \quad \text { (restricted to } X \text { ) }
$$

where $P, Q$ are homogeneous polynomials with $Q(x) \neq 0$ and apply the first observation.

Claim 2: Every element of $\mathcal{K}_{x}\left(\mathcal{O}_{x_{m}, x}\right)$ is a product of elements of the form

$$
\left\{f, g_{0}+g_{1} \epsilon+\cdots+g_{m} \epsilon^{m}\right\}, \quad\left\{1+u \epsilon^{i}, 1+v \epsilon^{j}\right\}
$$

where $f, g_{i}, u, v \in \mathcal{O}_{X, x}$.

Proof: We will show that for any $k$ with $0<k \leqq m$

$$
1+f_{k} \epsilon^{k}+f_{k+1} \epsilon^{k+1}+\cdots+f_{m} \epsilon^{m}=\left(1+f_{k} \epsilon^{k}\right)\left(1+f_{k+1} \epsilon^{k+1}+\tilde{f}_{k+2} \epsilon^{k+2}+\cdots+\tilde{f}_{m} \epsilon^{m}\right) .
$$

Assuming this we have

$$
f_{0}+f_{1} \epsilon+\cdots+f_{m} \epsilon^{m}=f_{0}\left(1+u_{1} \epsilon\right)\left(1+u_{2} \epsilon^{2}\right) \cdots\left(1+u_{m} \epsilon^{m}\right)
$$

for some $u_{1}, \ldots, u_{m}$, from which it follows that

$$
\begin{aligned}
\left\{f_{0}\right. & \left.+f_{1} \epsilon+\cdots+f_{m} \epsilon^{m}, g_{0}+g_{1} \epsilon+\cdots+g_{m} \epsilon^{m}\right\} \\
& =\left\{f_{0}\left(1+u_{1} \epsilon\right)\left(1+u_{2} \epsilon^{2}\right) \cdots\left(1+u_{m} \epsilon^{m}\right), g_{0}\left(1+v_{1} \epsilon\right)\left(1+v_{2} \epsilon^{2}\right) \cdots\left(1+v_{m} \epsilon^{m}\right)\right\} \\
& =\left\{f_{0}, g_{0}\right\} \prod_{i}\left\{f_{0}, 1+v_{i} \epsilon^{i}\right\} \prod_{j}\left\{1+u_{j} \epsilon^{j}, g_{0}\right\} \prod_{k, l}\left\{1+u_{k} \epsilon^{k}, 1+v_{l} \epsilon^{l}\right\}
\end{aligned}
$$


which proves the claim.

As for (3(ii).4) we have

$$
\left(1+f_{k} \epsilon^{k}\right)^{-1}=1-f_{k} \epsilon^{k}+f_{k}^{2} \epsilon^{2 k}-\cdots
$$

so that

$$
\left(1+f_{k} \epsilon^{k}\right)^{-1}\left(1+f_{k} \epsilon^{k}+f_{k+1} \epsilon^{k+1}+\cdots\right)=1+f_{k+1} \epsilon^{k+2} \bmod \epsilon^{k+2}
$$

which gives (3(ii).4). 
Claim 3: This is the same as claim 2, but where now we only use elements of the form

$$
\left\{1+u^{i} \epsilon^{i}, 1+v^{j} \epsilon^{j}\right\} \quad u, v \in \mathcal{O}_{X, x}
$$

in the second factors.

Proof: Using claim 1 to write

$$
\left\{\begin{array}{l}
u=p_{1}^{i}+\cdots+p_{k}^{i} \\
v=q_{1}^{j}+\cdots+q_{l}^{j}
\end{array}\right.
$$

we have

$$
\prod_{\alpha, \beta}\left\{1+p_{\alpha}^{i} \epsilon^{i}, 1+q_{\beta}^{j} \epsilon^{j}\right\}=\left\{1+u^{i} \epsilon^{i}, 1+v^{j} \epsilon^{j}\right\} \cdot T
$$

where $T$ is a product of terms of the form

$$
\left\{1+u^{\prime} \epsilon^{i^{\prime}}, 1+v^{\prime} \epsilon^{j^{\prime}}\right\}
$$

where

$$
i^{\prime}+j^{\prime}>i+j .
$$

Now we use descending induction on $i+j$, the case $i+j \geqq 2 m+1$ being automatic.

Claim 4: If $\omega_{1}, \ldots, \omega_{i}$ are the $i^{\text {th }}$ roots of -1 and $\eta_{1}, \ldots, \eta_{j}$ the $j^{\text {th }}$ roots of -1 , then

$$
\prod_{\alpha, \beta}\left\{1+\omega_{\alpha} u \epsilon, 1+\eta_{\beta} v \epsilon\right\}=\left\{1+u^{i} \epsilon^{i}, 1+v^{j} \epsilon^{j}\right\} .
$$

Proof: This follows from

$$
\begin{aligned}
& \prod_{\alpha=2}^{i}\left(1+\omega_{\alpha} u \epsilon\right)=1+u^{i} \epsilon^{i} \\
& \prod_{\beta=1}^{j}\left(1+\eta_{\beta} v \epsilon\right)=1+v^{j} \epsilon^{j} .
\end{aligned}
$$

Claim 5: If $u(x) \neq v(x)$, then

$$
\{1+u \epsilon, 1+v \epsilon\}\left\{\frac{v}{v-u}, 1+v \epsilon\right\}\left\{1+u \epsilon, \frac{u}{u-v}\right\}=1 .
$$

Proof: If $A$ and $A-1 \in \mathcal{O}_{X, x}^{*}$, then $A+B \epsilon \in \mathcal{O}_{X_{1}, x}^{*}$ and by the Steinberg relation

$$
\{A+B \epsilon, 1-A-B \epsilon\}=1 \text {. }
$$

If follows that

$$
1=\{A, 1-A\}\left\{A, 1-\frac{B \epsilon}{1-A}\right\}\left\{1+\frac{B \epsilon}{A}, 1-A\right\}\left\{1+\frac{B \epsilon}{A}, 1-\frac{B \epsilon}{1-A}\right\} .
$$

Setting

$$
u=B / A, \quad v=B / A-1,
$$

i.e.

$$
A=v /(v-u), \quad 1-A=u /(u-v)
$$


then

$$
1=\left\{\frac{v}{v-u}, 1+v \epsilon\right\}\left\{1+u \epsilon, \frac{u}{u-v}\right\}\{1+u \epsilon, 1+v \epsilon\} .
$$

In our arguments we will always be able to arrange that $u(x) \neq v(x)$.

Proof of proposition (3(ii).3): We first write

$$
u=p_{1}^{i}+\cdots+p_{k}^{i}, \quad v=q_{1}^{j}+\cdots q_{l}^{j}
$$

where, using the notations from Claim 3 ,

$$
\omega_{\alpha} p_{\lambda}(x) \neq \eta_{\beta} q_{\mu}(x) \quad \text { for all } \alpha, \beta, \lambda, \mu .
$$

This is easy to arrange. Then

$$
\left\{1+u \epsilon^{i}, 1+v \epsilon^{j}\right\} \equiv \prod_{\alpha, \beta, \lambda, \mu}\left\{1+\omega_{\alpha} p_{\lambda} \epsilon, 1+\eta_{\beta} q_{\mu} \epsilon\right\}
$$

modulo terms of the form $\left\{1+u^{\prime} \epsilon^{i^{\prime}}, 1+v^{\prime} \epsilon^{j^{\prime}}\right\}$ with $i^{\prime}+j^{\prime}>i+j$, and

$$
\begin{aligned}
& \left\{1+\omega_{\alpha} p_{\lambda} \epsilon, 1+\eta_{\beta} q_{\mu} \epsilon\right\} \\
& =\left\{1+\eta_{\beta} q_{\mu} \epsilon, \frac{\eta_{\beta} q_{\mu}}{\eta_{\beta} q_{\mu}-\omega_{\alpha} p_{\lambda}}\right\}\left\{\frac{\omega_{\alpha} p_{\lambda}}{\omega_{\alpha} p_{\lambda}-\eta_{\beta} q_{\mu}}, 1+\omega_{\alpha} p_{\lambda} \epsilon\right\} .
\end{aligned}
$$

This completes the proof: Claim 2 gets us down to elements in the statement of the proposition and terms of the forms $\left\{1+u \epsilon^{i}, 1+\epsilon v^{j}\right\}$. Claim 3 gets us down to elements we want plus terms $\left\{1+u^{i} \epsilon^{i}, 1+v^{j} \epsilon^{j}\right\}$. Claim 4 reduces these to products of $\{1+A \epsilon, 1+B \epsilon\}$ terms. Finally, the $\{1+A \epsilon, 1+B \epsilon\}$ terms are written as products of the form

$$
\{f, 1+A \epsilon\} \text { and }\{1+A \epsilon, f\}
$$

which have the desired form.

From (3(i).6) (iii) ${ }_{p}$ and (3(ii.1) we infer the commutative diagram (3(ii).5)

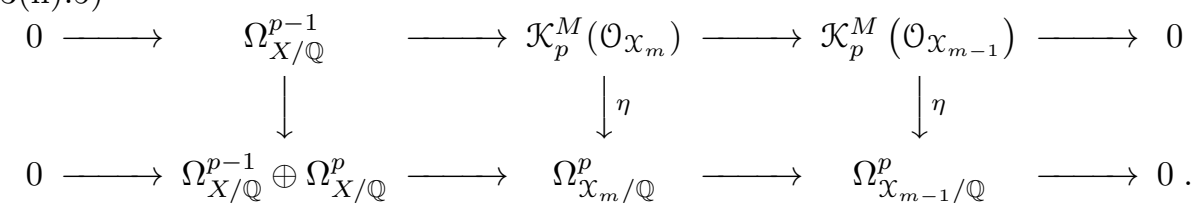

Here the left hand vertical arrow is

$$
\varphi \longrightarrow \varphi \oplus d \varphi
$$

and the other two vertical arrows are the $\wedge^{p} d \log$ maps. We recall that the bottom left horizontal arrow is

$$
\gamma \oplus \omega \rightarrow m t^{m-1} d t \wedge \gamma+t^{m} \omega
$$

and the commutativity of (3(ii).5) follows from (3(ii).2). From proposition (3(i).10) we deduce the

(3(ii).6) Proposition: The obstruction to lifting $\xi \in H^{p}\left(\mathcal{K}_{p}^{M}\left(\mathcal{O}_{x_{m-1}}\right)\right.$ ) to $H^{p}\left(\mathcal{K}_{p}^{M}\left(\mathcal{O}_{X_{m}}\right)\right)$ is given by

$$
\left.\delta(\xi)=\theta_{m}\right\rfloor \eta(\xi)
$$


where $\theta_{m}$ is the $m^{\text {th }}$ order Kodaira-Spencer class and $\eta(\xi)$ is the arithmetic cycle class of $\xi$.

(3(ii).7) Corollary: Let $\xi \in C H^{p}(X)$ and suppose that the fundamental class $\psi_{0}(\xi)$ remains of Hodge type $(p, p)$ in an analytic neighborhood of $t_{0} \in B$. Assume also that

$$
H^{p+1}\left(\Omega_{X / \mathbb{C}}^{p-2}\right)=\cdots=H^{p+1}\left(\mathcal{O}_{X}\right)=0 .
$$

Then $\xi$ lifts formally to $H^{p}\left(\mathcal{K}_{p}^{M}\left(\mathcal{O}_{\hat{x}}\right)\right.$.

Remark: If (3(ii).8) is not satisfied, then the HC implies that we may find $\xi^{\prime} \in$ $F^{1} C H^{p}(X)$ such that $\xi+\xi^{\prime}$ lifts to $H^{p}\left(\mathcal{K}_{p}^{M}\left(\mathcal{O}_{x}\right)\right.$ ) (and hence to $H^{p}\left(\mathcal{K}_{p}^{M}\left(\mathcal{O}_{\hat{x}}\right)\right)$ ). From (3(i).14) we know the corresponding statement for the arithmetic cycle class $\eta(\xi)$; we do not know that we can choose

$$
\eta^{\prime}=\eta\left(\xi^{\prime}\right)
$$

for some $\xi^{\prime} \in F^{1} C H^{p}(X)$; in fact, this is a geometric existence theorem that would follow from the HC applied to the spread of $X$ and $\xi$ (cf. (2(ii).23) - there are evident higher order analogues of this statement).

(3(ii.9) Corollary: Any $\xi \in F^{p} C H^{p}(X)$ formally lifts to $H^{p}\left(\mathcal{K}_{p}^{M}\left(\mathcal{O}_{\hat{x}}\right)\right)$.

Proof: This is just the higher order extension of (2(ii).24).

We now turn to the more arithmetic aspects and shall prove the following

(3(ii).10) Proposition: Suppose that $X$ is defined over $\mathbb{Q}$ and that $\xi \in C H^{p}(X(\mathbb{Q}))$. Suppose further that the fundamental class $\psi_{0}(\xi)$ remains of Hodge type $(p, p)$ in all $H^{2 p}\left(X_{t}, \mathbb{C}\right)$ for $t$ in an analytic neighborhood of $t$. Then $\xi$ lifts formally to $H^{p}\left(\mathcal{K}_{p}^{M}\left(\mathcal{O}_{\hat{x}}\right)\right)$.

Proof: It will simplify the notation to take the case $p=2$; the general argument is the same. Since $X$ is defined over $\mathbb{Q}$, the sequence

$$
0 \longrightarrow \Omega_{\mathbb{C} / \mathbb{Q}}^{1} \otimes \mathcal{O}_{X} \longrightarrow \Omega_{X / \mathbb{Q}}^{1} \longrightarrow \Omega_{X / \mathbb{C}}^{1} \longrightarrow 0
$$

splits as a sequence of $\mathcal{O}_{X}$-modules (see below). Making as usual the identification $C H^{2}(X) \cong H^{2}\left(\mathcal{K}_{2}\left(\mathcal{O}_{X}\right)\right)$, referring to (2(ii).11) the obstruction $\delta(\xi) \in H^{3}\left(\Omega_{X / \mathbb{Q}}^{1}\right)$ to lifting $\xi$ to $H^{2}\left(\mathcal{K}_{2}\left(\mathcal{O}_{X_{1}}\right)\right)$ may, using the splitting of (3(ii).11), be written as

$$
\delta(\xi)=\mathcal{O}_{1}(\xi) \oplus \mathcal{O}_{2}(\xi) \in H^{3}\left(\Omega_{X / \mathbb{C}}^{1}\right) \oplus \Omega_{\mathbb{C} / \mathbb{Q}}^{1} \otimes H^{3}\left(\mathcal{O}_{X}\right) .
$$

The primary $1^{\text {st }}$ order obstruction $\mathcal{O}_{1}(\xi)$ vanishes by our assumption on $\psi_{0}(\xi)$, and therefore we have to show that the secondary $1^{\text {st }}$ order obstruction

$$
\mathcal{O}_{2}(\xi)=0 \text {. }
$$

Geometrically, this is clear from the interpretation of $\mathcal{O}_{2}(\xi)$, the reason being that the spread of the pair $(X, \xi)$ has no continuous parameters. However, we want to give a computational proof that will extend to higher order.

Proof: We begin with some background remarks. Let $x_{1}, \ldots x_{n} \in \mathbb{Q}(X)$ give local uniformizing parameter in a Zariski neighborhood on $X$. Denote by $d f \in \Omega_{X / \mathbb{Q}, x}^{1}$ 
the absolute Kähler differential of $f \in \mathcal{O}_{X, x}$, and by $d_{\mathbb{C}} f$ the image of $d f$ in $\Omega_{X / \mathbb{C}, x}^{1}$. The map

$$
d_{\mathbb{C}} x_{i} \longrightarrow d x_{i}
$$

extended by $\mathcal{O}_{X, x}$-linearity splits the sequence (3(ii).11).

Locally in the analytic topology, any $f \in \mathcal{O}_{X, x}$ may be expanded as a power series in the $x_{i}$

$$
f=\sum_{I} a_{I} x^{I} \quad\left(x^{I}=\left(x_{1}\right)^{i_{1}} \cdots\left(x_{n}\right)^{i_{n}}\right) .
$$

If $f \in \mathcal{O}_{X(k), x}$ for some field $k$ with $\mathbb{Q} \subset k \subset \mathbb{C}$, then the $a_{I} \in k$. As was proved in [G-G2], we may compute the absolute differential $d f$ by differentiating the series (3(ii).14) term by term, where $d a_{I} \in \Omega_{k / \mathbb{Q}}^{1}$ is the absolute Kähler differential of $a_{I} \in k$. The resultant series converges and represents the power series expansion of $d f$. If $k=\overline{\mathbb{Q}}$, then the $d a_{I}=0$ and $d f$ is given by termwise differentiation of (3(ii).14) in the usual sense. As a consequence, if $f \in \mathcal{O}_{X, x} \cap \mathbb{Q}(X)$ and

$$
d_{\mathbb{C}} f=\sum_{i} f_{i} d_{\mathbb{C}} x_{i}
$$

where $f_{i} \in \mathcal{O}_{X, x} \cap \mathbb{Q}(X)$, then it follows that

$$
d f=\sum_{i} f_{i} d x_{i}
$$

This implies that the splitting of (3(ii).11) given by (3(ii).13) is independent of the choice of local uniformizing parameters.

Now let $\left\{U_{\alpha}\right\}$ be a Zariski covering of $X$ and let

$$
\left\{\begin{array}{l}
\xi \in H^{2}\left(\mathcal{K}_{2}\left(\mathcal{O}_{X(k)}\right)\right) \text { be represented by }\left\{g_{\alpha \beta \gamma}, h_{\alpha \beta \gamma}\right\} \\
\theta \in H^{1}\left(\Theta_{X / \mathbb{C}}\right) \text { be represented by } \theta_{\alpha \beta} \partial / \partial x_{\alpha} .
\end{array}\right.
$$

Then

$$
\left.\delta(\xi)_{\alpha \beta \gamma \delta}=\theta_{\alpha \beta}\right\rfloor\left(\frac{d g_{\beta \gamma \lambda}}{g_{\beta \gamma \lambda}} \wedge \frac{d h_{\beta \gamma \lambda}}{h_{\beta \gamma \lambda}}\right) .
$$

If $g_{\beta \gamma \lambda}$ and $h_{\beta \gamma \lambda}$ are represented by series as in (3(ii).14) above, then the $\mathcal{O}_{2}(\xi)_{\alpha \beta \gamma \lambda}$ component of $\delta(\xi)_{\alpha \beta \gamma \lambda}$ is represented by the series

$$
\left.\theta_{\alpha \beta}\right\rfloor\left\{\frac{\bar{d} g_{\beta \gamma \lambda}}{g_{\beta \gamma \lambda}} \wedge \frac{d_{\mathbb{C}} h_{\beta \gamma \lambda}}{h_{\beta \gamma \lambda}}+\frac{d_{\mathbb{C}} g_{\beta \gamma \lambda}}{g_{\beta \gamma \lambda}} \wedge \frac{\bar{d} h_{\beta \gamma \lambda}}{h_{\beta \gamma \lambda}}\right\}
$$

where in (3(ii).14) we have set

$$
\bar{d} f=\sum_{I} d a_{I} x^{I} \in \Omega_{k / \mathbb{Q}}^{1} \otimes \mathcal{O}_{X, x} .
$$

In particular, if $k=\mathbb{Q}$ then $\bar{d} g_{\beta \gamma \lambda}=\bar{d} h_{\beta \gamma \lambda}=0$ and so $\mathcal{O}_{2}(\xi)=0$, thereby establishing (3(ii).12).

If $\xi \in H^{2}\left(\mathcal{K}_{2}\left(\mathcal{O}_{X(\overline{\mathbb{Q}})}\right)\right.$ and $\mathcal{O}_{1}(\xi)=0$, then $\delta(\xi)=0$ and we may write the obstruction cocycle (3(ii).15) as a coboundary of a cochain defined over $\mathbb{Q}$. Thus we may lift $\xi$ to

$$
\left\{g_{\alpha \beta \gamma}+t g_{\alpha \beta \gamma}^{\prime}, h_{\beta \gamma \lambda}+t h_{\beta \gamma \lambda}^{\prime}\right\}, \quad t^{2}=0
$$


where $g_{\alpha \beta \gamma}^{\prime} \mathcal{O}_{X(\mathbb{Q})}\left(U_{\alpha \beta \gamma}\right)$ and $h_{\beta \gamma \lambda}^{\prime} \in \mathcal{O}_{X(\mathbb{Q})}\left(U_{\beta \gamma \lambda}\right)$. The fact that we may keep things defined over $\mathbb{Q}$ through $1^{\text {st }}$ order implies that when we calculate the $2^{\text {nd }}$ order obstruction to lifting $\xi$ only the deviation of $\psi_{0}(\xi)$ remaining of Hodge type $(2,2)$ to $2^{\text {nd }}$ order will appear. This process, when iterated, will lead to a proof of (3(ii).10).

We will discuss the next case of extending $\xi_{1} \in H^{2}\left(\mathcal{K}_{2}\left(\mathcal{O}_{X_{1}}\right)\right)$ given by (3(ii).16) to $\xi_{2} \in H^{2}\left(\mathcal{K}_{2}\left(\mathcal{O}_{X_{2}}\right)\right)$. By proposition (3(ii).6) the obstruction is given by

$$
\left.\delta\left(\xi_{1}\right)=\theta_{2}\right\rfloor \eta\left(\xi_{1}\right) \in H^{3}\left(\Omega_{X / \mathbb{Q}}^{1}\right) .
$$

Using the splitting of (3(ii).11) we may write

$$
\delta\left(\xi_{1}\right)=\mathcal{O}_{1}\left(\xi_{1}\right) \oplus \mathcal{O}_{2}\left(\xi_{1}\right)
$$

where

$$
\left\{\begin{array}{l}
\left.\mathcal{O}_{1}\left(\xi_{1}\right)=\theta_{2}\right\rfloor \eta_{\mathbb{C}}\left(\xi_{1}\right) \in H^{3}\left(\Omega_{X / \mathbb{C}}^{1}\right) \\
\mathcal{O}_{2}\left(\xi_{1}\right) \in \Omega_{\mathbb{C} / \mathbb{Q}}^{1} \otimes H^{3}\left(\mathcal{O}_{X}\right) .
\end{array}\right.
$$

Now $\theta_{1}\left(\xi_{1}\right)$ represents the deviation of $\psi_{0}(\xi)$ remaining of Hodge type $(2,2)$ to $2^{\text {nd }}$ order, and by assumption it vanishes. As for $\mathcal{O}_{2}\left(\xi_{1}\right)$, it is computed from terms of the type

$$
\bar{d} \log \left(g_{\alpha \beta \gamma}+t f_{\alpha \beta \gamma}^{\prime}\right) \wedge d \log \left(h_{\beta \gamma \lambda}+t h_{\beta \gamma \lambda}^{\prime}\right) .
$$

More precisely, by (3(ii).17) a Cech representative of $\mathcal{O}_{2}\left(\xi_{1}\right)$ will be given by the contraction of (3(ii).18) with the vector field

$$
\left(\frac{1}{t}\right) \tilde{\theta}_{1 \alpha \beta} \partial / \partial x_{\alpha}+\tilde{\theta}_{2 \alpha \beta} \partial / \partial x_{\alpha}
$$

where the first term contracts the coefficient of $t$ in (3(ii).8) and the second contracts the other term. Since $g_{\alpha \beta \gamma}$ and $g_{\alpha \beta \gamma}^{\prime}$ are defined over $\mathbb{Q}$ this contraction vanishes.

Since the cocycles representing the cohomology classes that arise as in (3(ii).17) are only defined up to coboundaries, the conclusion that we may draw is that the Cech cocycle representing $\delta\left(\xi_{1}\right)$ is a coboundary, and as before since the vector field (3(ii).19) is defined over $\mathbb{Q}$ this coboundary may be taken to also be defined over $\mathbb{Q}$.

The argument now proceeds inductively over the infinitesimal neighborhoods $x_{m}$, taking care to ensure at each state that the coefficients in the formal power series remain in $\mathbb{Q}$.

\section{FAILURES OF THE FORMAL THEORY}

In classical algebraic geometry - where "classical" in the study of cycles refers to codimension one - it is a general principle that a construction that can be made formally can be made geometrically; we abbreviate this by saying that

$$
\text { formal } \Rightarrow \text { actual. }
$$

For example, a result from the earliest days of deformation theory - valid in either the algebraic or analytic setting — states that if we are given a family

$$
\left\{X_{t}\right\}_{t \in B}
$$


where $h^{2}\left(\mathcal{O}_{X}\right)=0$, and given $[Z] \in C H^{1}(X)$ then

$$
\text { there is a family }\left[Z_{t}\right] \in C H^{1}\left(X_{t}\right) \text { which specializes to }[Z] \text { at } t=t_{0} \text {. }
$$

Here there are two caveats: First, we are not saying that the cycle $Z$ deforms; only that its class in $C H^{1}(X)$ does. The second is that in the algebraic case we may have to pass an étalé covering of $B$. Finally, the assumption $h^{2}\left(\mathcal{O}_{X}\right)=0$ may be replaced by the assumption that the fundamental class $\psi_{0}(Z)$ remains of Hodge type $(1,1)$ in $H^{2}\left(X_{t}, \mathbb{C}\right)$ for $t$ in an analytic neighborhood of $t_{0}$.

In the analytic setting, the proof of this result may be done by showing inductively that the successive terms in the power series construction of $\xi_{t} \in H^{1}\left(\mathcal{K}_{1}\left(\mathcal{O}_{X_{t}}\right)\right)$ may be chosen in a "bounded" fashion so as to insure convergence. Such arguments are common in the works of Kodaira-Spencer, Grauert and others during the 1950's and 1960's.

However, in order to use the Bloch-Quillen result, we need our formal deformation to lift to a rational/algebraic function on $B$. Here, there is no GAGA principle to rely on. There has been considerable progress made in recent years in approaching issues of rationality and algebraicity; see the Bourbaki talk of [CL].

An even more basic illustration of (4.1) is the statement

$$
\text { every tangent vector } \tau \in T C H^{1}(X) \text { is tangent to an arc in } C H^{1}(X) \text {. }
$$

Here, by $T C H^{1}(X)$ we mean the $p=1$ case of the formal tangent space

$$
T C H^{p}(X)=H^{p}\left(\Omega_{X / \mathbb{Q}}^{p-1}\right)
$$

to the Chow groups defined by Bloch [cf. B3]. The result (4.3) is more commonly expressed by saying that $C H^{1}(X)$ is representable and reduced. For reasons that will appear shortly, we have expressed it in the form (4.3).

The central point of this section is to explain the following:

$$
\begin{aligned}
& \text { For } p \geqq 2 \text { the principle (4.1) is false. In fact, already for } p=2 \text { the analogues } \\
& \text { of (4.2) and (4.3) are false. }
\end{aligned}
$$

This will be done by a series of examples, based mostly on our paper [G-G2] in which we defined for $p=1, n$ the tangent space $T Z^{p}(X)$ to the space $Z^{p}(X)$ of codimension- $p$ cycles on $X$, the subspace $T Z_{\text {rat }}^{p}(X)$ to the subgroup of cycles rationally equivalent to zero, and then we showed in some cases that

$$
T C H^{p}(X) \cong T Z^{p}(X) / T Z_{\text {rat }}^{p}(X)
$$

As discussed in loc. cit we expect that these definitions can be extended for all $p$ and that (4.6) will hold in general. Also, as discussed in $\S 10$ we expect that essentially for the geometric reasons stated there - it can be shown that

Both $T Z^{p}(X)$ and $T Z_{\text {rat }}^{p}(X)$ are formally reduced. 
This means that every tangent vector is tangent to a formal arc in $Z^{p}(X)$ and $Z_{\text {rat }}^{p}(X)$. We shall also show that

(4.8) The principal (4.1) is valid for $T Z^{1}(X)$ and $T Z_{\mathrm{rat}}^{1}(X)$, but is in general false for $p \geqq 2$.

In the following examples we will use the notations and terminology of [G-G2].

Example (i): In $C H^{2}\left(\mathbb{P}^{2}, T\right)$ we consider the arc

$$
z(t)=(b, t)-(b, 1)-(1, t)+(1,1)
$$

where $b \in \bar{Q}^{*}, b$ not a root of unity. This arc is non-constant in $C H^{2}\left(\mathbb{P}^{2}, T\right)$, but its velocity

$$
z^{\prime}(t)=\frac{d b}{b} \in \Omega_{\mathbb{C} / \mathbb{Q}}^{1} \cong T G r^{2} C H^{2}\left(\mathbb{P}^{2}, T\right)
$$

is identically zero. In this, building a formal power series $F(t)$ in

$$
(Y, Y \cap T) \underset{Y \subset\left(\mathbb{P}^{2}, T\right)}{\oplus} \mathbb{C}(Y, Y \cap T)^{*}
$$

which maps to $z(t)$ under the map

$$
(Y, f) \mapsto \operatorname{div} f
$$

involves inductively solving in $\mathbb{C}^{*} \otimes_{\mathbb{Z}} \mathbb{C}^{*}$ equations of the type

$$
\prod_{i=1}^{m} a_{i} \otimes b_{i}=\prod_{\nu=1}^{n}\left(1-c_{\nu}\right) \otimes c_{\nu}, \quad c_{\nu} \neq 1 .
$$

Here, the LHS is given and the RHS is to be solved for.

The assumption that $b$ is not a root of unity implies that $z(t)$ is non-constant. We are grateful to A. Merkurjev for explaining this. To solve for the terms in the series, at the first step the assumption that $b \in \bar{Q}^{*}$ gives

$$
d b=0 \quad\left(d=d_{\mathbb{C} / \mathbb{Q}}\right) .
$$

We can then solve the equation

$$
(1+t) \otimes b=\prod_{\nu=1}^{n}\left(a_{\nu}+t b_{\nu}\right) \otimes\left(1-a_{\nu}-t b_{\nu}\right), \quad t^{2}=0 \text { and } a_{\nu} \in \mathbb{Q}^{*} \backslash\{1\}, b_{\nu} \in \mathbb{Q},
$$

where the length $n$ of the Steinberg relation on the right depends on the arithmetic complexity of $b$. This gives the $t$-coefficient in the formal series for $F(t)$. For the $t^{2}$-coefficient we again have to an equation of this type where the length of the Steinberg relation depends on the arithmetic complexity of the $a_{\nu}$ and the $b_{\nu}$. There is no inductive choice of the solution to these equations that will yield a convergent answer. Although we don't know how to make this precise, we feel that the underlying reason is that there is no way to bound the arithmetic complexity of the successive choices. In any case, the above is an example of an $\operatorname{arc}$ in $Z^{2}\left(\mathbb{P}^{2}, T\right)$ whre $z^{\prime}(t) \in Z_{\text {rat }}^{2}\left(\mathbb{P}^{2}, T\right)$ but where there can only be formal arcs in (4.9) that map to $z(t)$.

Example (ii): Let $X$ be a regular surface with $H^{2}\left(\mathcal{O}_{X}\right) \neq 0, Y \subset X$ a smooth curve and suppose that $X$ and $Y$ are both defined over $\mathbb{Q}$ (or over a number field). Then the map

$$
C H^{1}(Y) \longrightarrow C H^{2}(X)
$$


is in general non-constant, while its differential

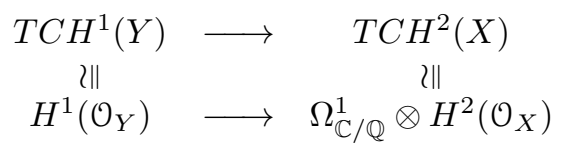

is identically zero. Although not as explicit, the reason seems to be analogous to that in example (i).

Example (iii): Let $X$ be a threefold defined over $\mathbb{Q}$ having $H^{2}\left(\mathcal{O}_{X}\right)=0$ and for which the abelian part $J_{\mathrm{ab}}^{2}(X)$ of the intermediate Jacobian $J^{2}(X)$ is zero - i.e., there are no sub-Hodge structures in $H^{2,1}(X) \oplus H^{1,2}(X)$ (for the existence of such see example (iv) below). Then (cf. [G-G2])

$$
T G r^{2} C H^{2}(X) \cong H^{2}\left(\Omega_{X / \mathbb{C}}^{1}\right)
$$

may be non-zero but, since $J_{\mathrm{ab}}^{2}(X)=0$ there are no actual families. In this case, for $\varphi \in H^{2}\left(\Omega_{X / \mathbb{C}}^{1}\right)$ there is a tangent vector

$$
\tau \in T Z^{2}(X)
$$

which maps to $\varphi$ under the isomorphism (4.6). Any arc in $Z^{2}(X)$ with tangent $\tau$ can only be formal; assuming the heurestic $(4.7), Z^{2}(X)$ is only formally reduced.

In concluding we would like to speculate on one possible way of understanding the convergence issues that arise above and in [G-G2].

In the classical case of deformation theory - e.g., the deformation of classes in $\mathrm{CH}^{1}(\mathrm{X})$ - the objects encountered are finite dimensional schemes (more formally, the functors are representable), and by results such as the Artin approximation theorem any construction that can be made formally can be modified at sufficiently high order to be convergent. Analytically we are inductively solving equations of the type

$$
\varphi_{i}=d \psi_{i}
$$

with sup norm estimates of the sort

$$
\left\|\psi_{i}\right\| \leqq C\left\|\varphi_{i}\right\|
$$

that are sufficient to obtain convergence in the analytic category.

In the non-classical case where the objects are not finite dimensional and arithmetic/geometric considerations arise - e.g., the deformation of classes in $C H^{P}(X)$ for $p \geqq 2$ - the issues of convergence are more subtle. One way of thinking of it is that we have to be able to construct convergent series expansions of algebraic functions defined over, say, $\mathbb{Q}$. To do so we have to be able to solve (4.10) with estimates (4.11) where we think of \|\| as ||$_{\infty}$, together with estimates

$$
\left|\psi_{i}\right|_{p} \leqq C\left|\varphi_{i}\right|_{p}
$$

for each prime $p$. We must bound "size" both in the usual analytic sense and in the sense of arithmetic complexity.

\section{REFERENCES}

[A] Y. André, G-functions and geometry, Aspects of Mathematics, vol. E13 (1989).

[B1] S. Bloch, Semi-regularity and deRham cohomology, Invent. Math. 17 (1972), 51-56.

[B2] S. Bloch, K and algebraic cycles, Ann. of Math. 99(2) (1974), 349-379.

[B3] S. Bloch, Lectures on algebraic cycles, Duke Univ. Math Ser. IV (1980), 182 pp. 
[C] H. Clemens, Cohomology and obstruction I: On the geometry of formal Kuranishi theory, to appear.

[CL] A. Chambert-Loir, Théorèmes d'algébricité en géométrie diophantienne, Sem. Bourbaki $\mathrm{n}^{\mathrm{O}} 886(2000-2001)$.

[EP] H. Esnault and K. Paranjape, Remarks on absolute deRham and absolute Hodge cycles (English. English, French summary), C. R. Acad. Sci. Paris Sér. I Math. 319(1) (194), $67-72$.

[EV] H. Esnault and E. Viehweg, Higher Kodaira-Spencer classes, Math. Ann. 299 (1994), 491-527.

[G] M. Green, Griffiths' infinitesimal invariant and the Abel-Jacobi map, J. Differential Geom. 29 (1989), 545-555.

[G-G1] M. Green and P. Griffiths, Hodge-theoretic invariants for algebraic cycles, to appear in IMRN.

[G-G2] M. Green and P. Griffiths, On the tangent space to the space of algebraic cycles on a smooth algebraic variety, to appear.

[R] Z. Ran, Derivatives of moduli, Internat. Math. Res. Notices no. 4 (1993),93-106.

[S] C. Soúle, , Opérations en SKS-théorie algébrique. (French) [Operations in algebraic SKStheory] Canad. J. Math. 37 (1985) no. 3, 488-550.

[Sv] V. Srinivas, Gysin maps and cycle classes for Hodge cohomology, Proc. Indian Acad. Sci. Math. Sci. 103 (1993), no. 3, 209-247.

Department of Mathematics, UCLA and Institute for Advanced Study 